\title{
FAMILIES OF ABSOLUTELY SIMPLE HYPERELLIPTIC JACOBIANS
}

\author{
YURI G. ZARHIN
}

\begin{abstract}
We prove that the jacobian of a hyperelliptic curve $y^{2}=(x-t) h(x)$ has no nontrivial endomorphisms over an algebraic closure of the ground field $K$ of characteristic zero if $t \in K$ and the Galois group of the polynomial $h(x)$ over $K$ is an alternating or symmetric group on $\operatorname{deg}(h)$ letters and $\operatorname{deg}(h)$ is an even number $>8$. (The case of odd $\operatorname{deg}(h)>3$ follows easily from previous results of the author.)
\end{abstract}

\section{Statements}

As usual, $\mathbb{Z}, \mathbb{Q}$ and $\mathbb{C}$ stand for the ring of integers, the field of rational numbers and the field of complex numbers respectively. If $\ell$ is a prime then we write $\mathbb{F}_{\ell}, \mathbb{Z}_{\ell}$ and $\mathbb{Q}_{\ell}$ for the $\ell$-element (finite) field, the ring of $\ell$-adic integers and field of $\ell$-adic numbers respectively. If $A$ is a finite set then we write $\#(A)$ for the number of its elements.

Let $K$ be a field of characteristic different from 2, let $\bar{K}$ be its algebraic closure and $\operatorname{Gal}(K)=\operatorname{Aut}(\bar{K} / K)$ its absolute Galois group. Let $n \geq 5$ be an integer, $f(x) \in K[x]$ a degree $n$ polynomial without multiple roots, $\mathfrak{R}_{f} \subset \bar{K}$ the $n$-element set of its roots, $K\left(\mathfrak{R}_{f}\right) \subset \bar{K}$ the splitting field of $f(x)$ and $\operatorname{Gal}(f)=\operatorname{Gal}\left(K\left(\Re_{f}\right) / K\right)$ the Galois group of $f(x)$ over $K$. One may view $\operatorname{Gal}(f)$ as a certain group of permutations of $\mathfrak{R}_{f}$. Let $C_{f}: y^{2}=f(x)$ be the corresponding hyperelliptic curve of genus $\lfloor(n-1) / 2\rfloor$. Let $J\left(C_{f}\right)$ be the jacobian of $C_{f}$; it is a $\lfloor(n-1) / 2\rfloor$-dimensional abelian variety that is defined over $K$. We write $\operatorname{End}\left(J\left(C_{f}\right)\right)$ for the ring of all $\bar{K}$ endomorphisms of $J\left(C_{f}\right)$. As usual, we write $\operatorname{End}^{0}\left(J\left(C_{f}\right)\right)$ for the corresponding (finite-dimensional semisimple) $\mathbb{Q}$-algebra $\operatorname{End}\left(J\left(C_{f}\right)\right) \otimes \mathbb{Q}$.

In $[40,42,44]$ the author proved the following statement.

Theorem 1.1. Suppose that $\operatorname{Gal}(f)$ is either the full symmetric group $\mathbf{S}_{n}$ or the alternating group $\mathbf{A}_{n}$. Assume also that either $\operatorname{char}(K) \neq 3$ or $n \geq 7$. Then $\operatorname{End}\left(J\left(C_{f}\right)\right)=\mathbb{Z}$. In particular, $J\left(C_{f}\right)$ is an absolutely simple abelian variety.

The aim of this note is to discuss the structure of $\operatorname{End}\left(J\left(C_{f}\right)\right)$ when $f(x)$ has a root in $K$ and the remaining degree $(n-1)$ factor of $f(x)$ has "large" Galois group over $K$.

Remark 1.2. Suppose that $t \in K$ is a root of $f(x)$. By the division algorithm, $f(x)=(x-t) h(x)$ with $t \in K$ and $h(x)$ a polynomial of degree $n-1$ with coefficients in $K$. Then $\mathfrak{R}_{f}$ is the disjoint union of singleton $\{t\}$ and the $(n-1)$-element set $\mathfrak{R}_{h}$ of roots of $h(x)$. Clearly, $K\left(\mathfrak{R}_{h}\right)=K\left(\mathfrak{R}_{f}\right)$ and $\operatorname{Gal}(h)=\operatorname{Gal}(f)$.

Our first result is the following statement. 
Theorem 1.3. Suppose that $n=\operatorname{deg}(f) \geq 6$ is even, $f(x)=(x-t) h(x)$ with $t \in K$ and $h(x) \in K[x]$. Suppose that $\operatorname{Gal}(h)$ is either the full symmetric group $\mathbf{S}_{n-1}$ or the alternating group $\mathbf{A}_{n-1}$. Assume also that either $\operatorname{char}(K) \neq 3$ or $n \geq 8$. Then $\operatorname{End}\left(J\left(C_{f}\right)\right)=\mathbb{Z}$. In particular, $J\left(C_{f}\right)$ is an absolutely simple abelian variety.

Proof. We have $n=2 g+2$ where $g$ is the genus of $C_{f}$ and $n-1=2 g+1=\operatorname{deg}(h)$. Let us consider the polynomials

$$
h_{1}(x)=h(x+t), h_{2}(x)=x^{n-1} h_{1}(1 / x) \in K[x] .
$$

They all have degree $n-1 \geq 5$; in addition, $n-1 \geq 7$ if $\operatorname{char}(K)=3$. We have

$$
\mathfrak{R}_{h_{1}}=\left\{\alpha-t \mid \alpha \in \mathfrak{R}_{h}\right\}, \mathfrak{R}_{h_{2}}=\left\{\frac{1}{\alpha-t} \mid \alpha \in \mathfrak{R}_{h}\right\} .
$$

This implies that

$$
K\left(\Re_{h_{2}}\right)=K\left(\Re_{h_{1}}\right)=K\left(\Re_{h}\right)
$$

and therefore

$$
\operatorname{Gal}\left(h_{2}\right)=\operatorname{Gal}\left(h_{1}\right)=\operatorname{Gal}(h) .
$$

In particular, $\operatorname{Gal}\left(h_{2}\right)=\mathbf{S}_{n-1}$ or $\mathbf{A}_{n-1}$.

Now the equation for $C_{f}$ may be written down as

$$
y^{2}=(x-t) h_{1}(x-t) .
$$

Dividing both sides of the latter equation by $(x-t)^{2(g+1)}$, we get

$$
\left[y /(x-t)^{g+1}\right]^{2}=(x-t)^{-(n-1)} h_{1}(x-t)=h_{2}(1 /(x-t)) .
$$

Now the standard substitution

$$
x_{1}=1 /(x-t), y_{1}=y /(x-t)^{g+1}
$$

establishes a birational $K$-isomorphism between $C_{f}$ and a hyperelliptic curve

$$
C_{h_{2}}: y_{1}^{2}=h_{2}\left(x_{1}\right) \text {. }
$$

Now the result follows readily from Theorem 1.1 applied to the polynomial $h_{2}(x)$.

The case of odd $n$ is more difficult.

Theorem 1.4. Suppose that $n=\operatorname{deg}(f) \geq 9$ is odd and $f(x)=(x-t) h(x)$ with $t \in K$ and $h(x) \in K[x]$. Suppose that $\operatorname{Gal}(h)$ is either the full symmetric group $\mathbf{S}_{n-1}$ or the alternating group $\mathbf{A}_{n-1}$. Then one of the following conditions holds.

(i) $\operatorname{End}^{0}\left(J\left(C_{f}\right)\right)$ is either $\mathbb{Q}$ or a quadratic field. In particular, $J\left(C_{f}\right)$ is an absolutely simple abelian variety.

(ii) $\operatorname{char}(K)>0$ and $J\left(C_{f}\right)$ is a supersingular abelian variety.

When the genus is at least 5, we may improve the result as follows.

Theorem 1.5. Suppose that $n=\operatorname{deg}(f) \geq 11$ is odd and $f(x)=(x-t) h(x)$ with $t \in K$ and $h(x) \in K[x]$. Suppose that $\operatorname{Gal}(h)$ is either the full symmetric group $\mathbf{S}_{n-1}$ or the alternating group $\mathbf{A}_{n-1}$. If $\operatorname{char}(K)=0$ then $\operatorname{End}\left(J\left(C_{f}\right)\right)=\mathbb{Z}$. 
Remark 1.6. If $K$ is finitely generated over $\mathbb{Q}$ and $h(x) \in K[x]$ is an arbitrary polynomial of positive even degree without multiple roots then for all but finitely many $t \in K$ the jacobian of the hyperelliptic curve $y^{2}=(x-t) h(x)$ is absolutely simple [7, Theorem 9]. (See also [18].) The authors of [7] use and compare approaches based on arithmetic geometry and analytic number theory respectively. In a sense, our approach is purely algebraic.

The paper is organized as follows. Section 2 contains auxiliary results from group theory. In Sections 3 and 4 we study the structure of endomorphism algebras of abelian varieties with certain Galois properties of points of order 2. Section 5 contains an explicit description of the Galois module of their points of order 2 on $\left.J\left(C_{f}\right)\right)$. Combining this description with results of Sections 3 and 4 , we prove Theorems 1.4 and 1.5. In Section 6 we discuss families of hyperelliptic curves. Section 7 contains auxiliary results about $\ell$-adic Lie groups and their Lie algebras. In Section 8 we prove under the conditions of Theorem 1.5 that if the ground field $K$ is finitely generated over $\mathbb{Q}$ then the image of $\operatorname{Gal}(K)$ in the automorphism group of the $\ell$-adic Tate module of $J\left(C_{f}\right)$ is almost "as large as possible", namely, it is an open subgroup in the group of symplectic similitudes. We use this openness property in order to prove for self-products of $J\left(C_{f}\right)$ the Tate and Hodge conjectures in Sections 9 and 10 respectively. Section 10 also contains the proof of the MumfordTate conjecture for $J\left(C_{f}\right)$.

I am grateful to the referee, whose comments helped to improve the exposition.

\section{Minimal covers and Representations of alternating groups}

Proposition 2.1. Let $m \geq 8$ be an integer, $\mathbf{A}_{m}$ the corresponding alternating group. Let $N$ be the smallest positive integer $d$ such that there exists a group embedding $\mathbf{A}_{m} \hookrightarrow \operatorname{PGL}(d, \mathbb{C})$. Then $N=m-1$.

Proof. First, (for all $m$ ) there exists a well-known group embedding $\mathbf{A}_{m} \hookrightarrow \operatorname{GL}(m-$ $1, \mathbb{C})$, which induces $\mathbf{A}_{m} \hookrightarrow \operatorname{PGL}(m-1, \mathbb{C})$. Let us consider the non-split short exact sequence of finite groups

$$
1 \rightarrow \mathbb{Z} / 2 \mathbb{Z} \hookrightarrow \mathbf{A}_{m}^{\prime} \rightarrow \mathbf{A}_{m} \rightarrow 1
$$

where $\mathbf{A}_{m}^{\prime}$ is the universal central extension of $\mathbf{A}_{m}$ and $\mathbb{Z} / 2 \mathbb{Z}$ is the center of $\mathbf{A}_{m}^{\prime}$. (Recall that $m \geq 8$.) Let $c$ be the only nontrivial element of the center of $\mathbf{A}_{m}^{\prime}$.

Now suppose that we are given a group embedding $\mathbf{A}_{m} \hookrightarrow \operatorname{PGL}(d, \mathbb{C})$. We need to prove that $d \geq m-1$. The universality property of $\mathbf{A}_{m}^{\prime}$ implies that the embedding is the projectivization of a (nontrivial) linear representation

$$
\rho^{\prime}: \mathbf{A}_{m}^{\prime} \hookrightarrow \mathrm{GL}(V)
$$

where $V=\mathbb{C}^{d}$. Replacing $V$ by its $\mathbf{A}_{m}^{\prime}$-invariant subspace of minimal dimension with nontrivial action of $\mathbf{A}_{m}^{\prime}$, we may and will assume that $\rho^{\prime}$ is a nontrivial irreducible (but not necessary faithful) representation of $\mathbf{A}_{m}^{\prime}$. We need to prove that $\operatorname{dim}_{\mathbb{C}}(V) \geq m-1$. Schur's Lemma implies that

$$
\rho^{\prime}(c) \in\{1,-1\} \subset \mathbb{C}^{*} .
$$

If $\rho^{\prime}(c)=1$ then $\rho^{\prime}$ factors through $\mathbf{A}_{m}$ and we get a nontrivial linear representation $\mathbf{A}_{m} \hookrightarrow \operatorname{GL}(V)$, which must be faithful in light of the simplicity of $\mathbf{A}_{m}$. If this is 
the case then $\operatorname{dim}_{\mathbb{C}}(V) \geq m-1$ [14, p. 71 , Theorem 2.5.15]. So, further, we may and will assume that

$$
\rho^{\prime}(c)=-1,
$$

and therefore $\rho^{\prime}$ is faithful, i.e., $\rho$ is a proper projective representation of $\mathbf{A}_{m}[36$, p. 584]. Now an old result of Schur [32, S. 250] (see also [36, Th. 1.3(ii)] and [17, Th. A on p. 1972]) asserts that $\operatorname{dim}_{\mathbb{C}}(V) \geq m-1$.

2.2. Recall [11] that a surjective homomorphism of finite groups $\pi: \mathcal{G}_{1} \rightarrow \mathcal{G}$ is called a minimal cover if no proper subgroup of $\mathcal{G}_{1}$ maps onto $\mathcal{G}$. In particular, if $\mathcal{G}$ is perfect and $\mathcal{G}_{1} \rightarrow \mathcal{G}$ is a minimal cover then $\mathcal{G}_{1}$ is also perfect. In addition, if $r$ is a positive integer such that every subgroup in $\mathcal{G}$ of index dividing $r$ coincides with $\mathcal{G}$ then the same is true for $\mathcal{G}_{1}$ [48, Remark 3.4]. Namely, every subgroup in $\mathcal{G}_{1}$ of index dividing $r$ coincides with $\mathcal{G}$.

Lemma 2.3. Let $m \geq 5$ be an integer, $\mathbf{A}_{m}$ the corresponding alternating group and $\mathcal{G}_{1} \rightarrow \mathbf{A}_{m}$ a minimal cover.

Then:

(i) The only subgroup of index $<m$ in $\mathcal{G}_{1}$ is $\mathcal{G}_{1}$ itself.

(ii) Suppose that $m \geq 8$. If $d$ is a positive integer such that there exists a group embedding $\mathcal{G}_{1} \hookrightarrow \operatorname{PGL}(d, \mathbb{C})$ then $d \geq m-1$.

Proof. Let $H$ be a subgroup in $\mathbf{A}_{m}$ of index $r>1$. Then $\mathbf{A}_{m}$ acts transitively on the $r$-element set of (left) $H$-cosets. Therefore there is a nontrivial homomorphism $\mathbf{A}_{m} \rightarrow \mathbf{S}_{r}$, which must be an embedding in light of the simplicity of $\mathbf{A}_{m}$. Comparing the orders, we conclude that

$$
r ! \geq \frac{m !}{2}>(m-1) !
$$

and therefore $r \geq m$. This implies that the only subgroup of index $<m$ in $\mathbf{A}_{m}$ is $\mathbf{A}_{m}$ itself. Now arguments of Sect. 2.2 imply that the only subgroup of index $<m$ in $\mathcal{G}_{1}$ is $\mathcal{G}_{1}$ itself. This proves (i).

Now assume that $m \geq 8$. By Proposition 2.1, if $d$ is a positive integer such that there exists a group embedding $\mathbf{A}_{m} \hookrightarrow \operatorname{PGL}(d, \mathbb{C})$ then $d \geq m-1$. Applying Theorem on p. 1092 and Proposition 4.1 (combined with Sect. 4.2) of [11], we conclude that if $d$ is a positive integer such that there exists a group embedding $\mathcal{G}_{1} \hookrightarrow \operatorname{PGL}(d, \mathbb{C})$ then $d \geq m-1$.

Remark 2.4. If $m \geq 10$ is an even integer then it follows from results of Wagner [35] that every projective representation of $\mathbf{A}_{m}$ in characteristic 2 has dimension $\geq m-2[42$, Remark 4.2].

Let $\mathcal{G}_{2} \rightarrow \mathbf{A}_{m}$ be a surjective homomorphism of finite groups. Suppose that $\mathbb{F}$ is a field of characteristic 2 and $d$ a positive integer such that there exists an embedding

$$
\mathcal{G}_{2} \hookrightarrow \operatorname{PGL}(d, \mathbb{F}) .
$$

I claim that $d \geq m-2$. Indeed, replacing $\mathcal{G}_{2}$ by its suitable subgroup, we may assume that $\mathcal{G}_{2} \rightarrow \mathbf{A}_{m}$ is a minimal cover. (E.g., one may take as $\mathcal{G}_{2}$ a subgroup of the smallest possible order that maps surjectively on $\mathbf{A}_{m}$.) Then the result follows from a theorem of Feit-Tits [11, p. 1092] (see also [16, Theorem 1]). 
Corollary 2.5. Suppose that $m \geq 10$ is an even integer, $L$ is a field that is a finite algebraic extension of $\mathbb{Q}_{2}$ and $V$ is a non-zero finite-dimensional vector space over $L$ with $d:=\operatorname{dim}_{L}(V)<m-2$. Let $G \subset \operatorname{Aut}_{L}(V)$ be a compact subgroup.

Then there does not exist a surjective continuous homomorphism $G \rightarrow \mathbf{A}_{m}$.

Proof. Suppose that there exists a surjective continuous homomorphism $\pi: G \rightarrow$ $\mathbf{A}_{m}$. We write $H$ for $\operatorname{ker}(\pi)$ : it is an open normal subgroup of finite index in $G$ and $G / H \cong \mathbf{A}_{m}$. The surjectivity of $\pi$ implies that the image of every normal subgroup of $G$ is normal in $\mathbf{A}_{m}$ and therefore is either $\{1\}$ or the whole $\mathbf{A}_{m}$.

Let $\mathcal{O}$ be the ring of integers in $L$. We write $\mathfrak{m}$ for the maximal ideal of $\mathcal{O}$ and $\mathbb{F}$ for the (finite) residue field $\mathcal{O} / \mathfrak{m}$. Notice that there exists a $G$-stable $\mathcal{O}$-lattice $T$ in $V$ of rank $d$. (Our proof of this assertion follows [30, Sect. 1.1].) Indeed, let $T^{\prime}$ be any $\mathcal{O}$-lattice in $V$ of rank $d$ and let $G^{\prime}$ be the set of $s \in G$ such that $s\left(T^{\prime}\right)=T^{\prime}$. This is an open subgroup of $G$, because $T^{\prime}$ is an open finitely generated

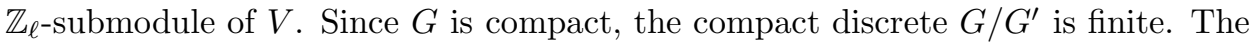
$\mathcal{O}$-lattice $T$ generated by the lattices $s\left(T^{\prime}\right), s \in G / G^{\prime}$, is $G$-stable. We have

$$
G \subset \operatorname{Aut}_{\mathcal{O}}(T) \subset \operatorname{Aut}_{L}(V) .
$$

We write $G_{0}$ for the kernel of the reduction map modulo $\mathfrak{m}$

$$
\operatorname{red}: G \rightarrow \operatorname{Aut}_{\mathcal{O}}(T / \mathfrak{m} T)
$$

and $\tilde{G}$ for its image. We have

$$
\tilde{G} \subset \operatorname{Aut}_{\mathcal{O}}(T / \mathfrak{m} T) \cong \mathrm{GL}(d, \mathbb{F}) .
$$

Clearly, $G_{0}$ is a pro-2-group and $\pi\left(G_{0}\right)$ is a normal 2-subgroup in $\mathbf{A}_{m}$. Since $\mathbf{A}_{m}$ is simple non-abelian, $\pi\left(G_{0}\right)=\{1\}$. This implies that $\pi$ factors through a surjective homomorphism

$$
\pi_{0}: \tilde{G}=G / G_{0} \rightarrow \mathbf{A}_{m} .
$$

The surjectivity of $\pi_{0}$ implies that the center of $\tilde{G}$ goes to the center of $\mathbf{A}_{m}$, i.e., $\pi_{0}$ kills the center of $\tilde{G}$; in particular $\pi_{0}$ kills the subgroup $\tilde{Z}$ of scalar matrices in $\tilde{G}$. This gives us the surjection $\tilde{G} / \tilde{Z} \rightarrow \mathbf{A}_{m}$ and the embedding $\tilde{G} / \tilde{Z} \hookrightarrow \operatorname{PGL}(d, \mathbb{F})$. It follows from Remark 2.4 that $d \geq m-2$, which is not the case and we get a desired contradiction.

2.6. Let $g \geq 3$ be an integer. Then $2 g \geq 6$ and $\mathbf{A}_{2 g}$ is a simple non-abelian group. Let $B$ be an $2 g$-element set. We write $\operatorname{Perm}(B)$ for the group of all permutations of $B$. The choice of ordering on $B$ establishes an isomorphism between $\operatorname{Perm}(B)$ and the symmetric group $\mathrm{S}_{2 g}$. We write $\operatorname{Alt}(B)$ for the only subgroup of index 2 in $\operatorname{Perm}(B)$. Clearly, every isomorphism $\operatorname{Perm}(B) \cong \mathrm{S}_{2 g}$ induces an isomorphism between $\operatorname{Alt}(B)$ and the alternating group $\mathbf{A}_{2 g}$. Let us consider the $2 g$-dimensional $\mathbb{F}_{2}$-vector space $\mathbb{F}_{2}^{B}$ of all $\mathbb{F}_{2}$-valued functions on $B$ provided with the natural structure of faithful Perm $(B)$-module. Notice that the standard symmetric bilinear form

$$
\mathbb{F}_{2}^{B} \times \mathbb{F}_{2}^{B} \rightarrow \mathbb{F}_{2},(\phi, \psi) \mapsto \sum_{b \in B} \phi(b) \psi(b)
$$

is non-degenerate and $\operatorname{Perm}(B)$-invariant.

Since $\operatorname{Alt}(B) \subset \operatorname{Perm}(B)$, one may view $\mathbb{F}_{2}^{B}$ as a faithful $\operatorname{Alt}(B)$-module.

Lemma 2.7. $\quad$ (i) The centralizer $\operatorname{End}_{\operatorname{Alt}(B)}\left(\mathbb{F}_{2}^{B}\right)$ has $\mathbb{F}_{2}$-dimension 2 . 
(ii) Every proper non-zero $\operatorname{Alt}(B)$-invariant subspace in $\mathbb{F}_{2}^{B}$ has dimension 1 or $2 g-1$. In particular, $\mathbb{F}_{2}^{B}$ does not contain a proper non-zero $\operatorname{Alt}(B)$ invariant even-dimensional subspace.

Proof. Since $\operatorname{Alt}(B)$ is doubly transitive, (i) follows from [22, Lemma 7.1].

Notice that the subspace of $\operatorname{Alt}(B)$-invariants

$$
M_{0}:=\left(\mathbb{F}_{2}^{B}\right)^{\operatorname{Alt}(B)}=\mathbb{F}_{2} \cdot 1_{B},
$$

where $1_{B}$ is the constant function 1 .

In order to prove (ii), recall that

$$
M_{0} \subset M_{1} \subset \mathbb{F}_{2}^{B}
$$

where $M_{1}$ is the hyperplane of functions with zero sum of values. It is known [19] that $M_{1} / M_{0}$ is a simple $\operatorname{Alt}(B)$-module; clearly, $\operatorname{dim}\left(M_{1} / M_{0}\right)=2 g-2$.

First, notice that there are no $\operatorname{Alt}(B)$-invariant two-dimensional $\mathbb{F}_{2}$-vector subspaces in $\mathbb{F}_{2}^{B}$. Indeed, let $W_{2}$ be an $\operatorname{Alt}(B)$-invariant $\mathbb{F}_{2}$-vector subspace in $\mathbb{F}_{2}^{B}$ with $\operatorname{dim}_{\mathbb{F}_{2}}\left(W_{2}\right)=2$. Since $\mathbf{A}_{2 g}$ is simple non-abelian and $\mathrm{GL}_{2}\left(\mathbb{F}_{2}\right)$ is solvable, every homomorphism

$$
\operatorname{Alt}(B) \rightarrow \operatorname{Aut}_{\mathbb{F}_{2}}\left(W_{2}\right) \cong \mathrm{GL}_{2}\left(\mathbb{F}_{2}\right)
$$

is trivial and therefore $W_{2}$ consists of $\operatorname{Alt}(B)$-invariants; however, the subspace of $\operatorname{Alt}(B)$-invariants in $\mathbb{F}_{2}^{B}$ is just one-dimensional.

Second, if $W$ is a $\operatorname{Alt}(B)$-invariant $(2 g-2)$-dimensional subspace of $\mathbb{F}_{2}^{B}$ then its orthogonal complement with respect to the standard form is a two-dimensional $\operatorname{Alt}(B)$-invariant subspace in $\mathbb{F}_{2}^{B}$. This implies that there are no $\operatorname{Alt}(B)$-invariant $(2 g-2)$-dimensional $\mathbb{F}_{2}$-vector subspaces in $\mathbb{F}_{2}^{B}$.

Let $W$ be a $\operatorname{Alt}(B)$-invariant subspace of $\mathbb{F}_{2}^{B}$ and assume that

$$
2<\operatorname{dim}_{\mathbb{F}_{2}}(W)<2 g-2 .
$$

This implies that the Alt $(B)$-invariant subspace

$$
W_{1}:=W \bigcap M_{1}
$$

is not $\{0\}$. Since $M_{1}$ is a hyperplane in $\mathbb{F}_{2}^{B}$, either $W=W_{1}$ or $\operatorname{dim}_{\mathbb{F}_{2}}(W)=$ $\operatorname{dim}_{\mathbb{F}_{2}}\left(W_{1}\right)+1$. If $W_{1}=M_{0}$ then

$$
\operatorname{dim}_{\mathbb{F}_{2}}(W) \leq \operatorname{dim}_{\mathbb{F}_{2}}\left(M_{0}\right)+1=2,
$$

which could not be the case. If $W_{1}=M_{1}$ then

$$
\operatorname{dim}_{\mathbb{F}_{2}}(W) \geq \operatorname{dim}_{\mathbb{F}_{2}}\left(M_{1}\right)=2 g-1,
$$

which also could not be the case. This implies that

$$
W_{1} \neq M_{0}, W_{1} \neq M_{1} \text {. }
$$

Since $M_{0}$ is a one-dimensional subspace of $M_{1}$, either $W_{1} \supset M_{0}$ or $W_{1} \cap M_{0}=$ $\{0\}$.

In the former case, $W_{1} / M_{0}$ is an $\operatorname{Alt}(B)$-invariant subspace of $M_{1} / M_{0}$ and the simplicity of $M_{1} / M_{0}$ implies that either $W_{1} / M_{0}=\{0\}$, i.e., $W_{1}=M_{0}$ or $W_{1} / M_{0}=M_{1} / M_{0}$, i.e., $W_{1}=M_{1}$. Since $W_{1}$ is neither $M_{0}$ nor $M_{1}$, we conclude that $W_{1} \cap M_{0}=\{0\}$. We are going to arrive to a contradiction. The natural map $W_{1} \rightarrow M_{1} / M_{0}$ is an embedding, whose image is a non-zero $\operatorname{Alt}(B)$-invariant 
subspace of $M_{1} / M_{0}$; the simplicity of the Alt(B)-module $M_{1} / M_{0}$ implies that the image of $W_{1}$ coincides with the whole $M_{1} / M_{0}$; in particular,

$$
\operatorname{dim}_{\mathbb{F}_{2}}\left(W_{1}\right)=\operatorname{dim}_{\mathbb{F}_{2}}\left(M_{1} / M_{0}\right)=2 g-2,
$$

and we get the $(2 g-2)$-dimensional $\operatorname{Alt}(B)$-invariant subspace, which could not exist. We get the desired contradiction.

Theorem 2.8. Let $g \geq 3$ be an integer, $B$ a $2 g$-element set, $V$ a $2 g$-dimensional $\mathbb{Q}_{2}$-vector space, $T$ a $\mathbb{Z}_{2}$-lattice in $V$ of rank $2 g$. Suppose that

$$
G \subset \operatorname{Aut}_{\mathbb{Z}_{2}}(T) \subset \operatorname{Aut}_{\mathbb{Q}_{2}}(V)
$$

is a compact (in the 2-adic topology) subgroup. Let

$$
\tilde{G}:=\operatorname{red}(G) \subset \operatorname{Aut}_{\mathbb{F}_{2}}(T / 2 T)
$$

be the image of $G$ with respect to the reduction map modulo 2

$$
\text { red }: \operatorname{Aut}_{\mathbb{Z}_{2}}(T) \rightarrow \operatorname{Aut}_{\mathbb{F}_{2}}(T / 2 T) .
$$

Suppose that there exists a group isomorphism $\tilde{G} \cong \operatorname{Alt}(B)$ such that the $\operatorname{Alt}(B)$ module $T / 2 T$ is isomorphic to $\mathbb{F}_{2}^{B}$.

Then:

(i) Every proper non-zero $G$-invariant subspace of $V$ has dimension either 1 or $2 g-1$.

(ii) Assume that $g \geq 5$. Let $\operatorname{End}_{G}(V)$ be the centralizer of $G$ in $\operatorname{End}_{\mathbb{Q}_{2}}(V)$. Suppose that $D$ is a semisimple commutative $\mathbb{Q}_{2}$-(sub)algebra of $\operatorname{End}_{G}(V)$ (with the same identity element) such that the $D$-module $V$ is free. Then $D=\mathbb{Q}_{2}$, i.e., $D$ consists of scalars.

Proof. The reduction map modulo 2

$$
\text { red : } \operatorname{Aut}_{\mathbb{Z}_{2}}(T) \rightarrow \operatorname{Aut}_{\mathbb{F}_{2}}(T / 2 T)=\operatorname{Aut}_{\mathbb{F}_{2}}\left(\mathbb{F}_{2}^{B}\right)
$$

induces a surjective continuous homomorphism

$$
\pi: G \rightarrow \tilde{G}=\operatorname{Alt}(B) .
$$

In order to prove (i), let us assume that there exists a $G$-invariant proper nonzero subspace $V_{1} \subset V$ and put $T_{1}:=V_{1} \cap T$. Clearly, $T_{1}$ is a $G$-invariant free $\mathbb{Z}_{2}$-submodule of $T$, the quotient $T / T_{1}$ is a torsion-free $\mathbb{Z}_{2}$-module and the $\mathbb{Z}_{2}$-rank of $T_{1}$ coincides with the $\mathbb{Q}_{2}$-dimension of $V_{1}$. Now, $T_{1} / 2 T_{1}$ is $\tilde{G}=\operatorname{Alt}(B)$-invariant subspace in $T / 2 T=\mathbb{F}_{2}^{B}$, whose $\mathbb{F}_{2}$-dimension coincides with the rank of $T$, i.e., with the $\mathbb{Q}_{2}$-dimension of $V_{1}$. It follows from Lemma 2.7 (ii) that $\operatorname{dim}_{\mathbb{F}_{2}}\left(T_{1} / 2 T_{1}\right)=1$ or $2 g-1$. It follows that $\operatorname{dim}_{\mathbb{Q}_{2}}\left(V_{1}\right)=1$ or $2 g-1$.

In order to prove (ii), first notice that $2 g \geq 10$. Let $h$ be the rank of the free $D$-module $V$ and $e=\operatorname{dim}_{\mathbb{Q}_{2}}(D)$. Clearly,

$$
2 g=\operatorname{dim}_{\mathbb{Q}_{2}}(V)=e h ;
$$

in particular, $e \mid 2 g$ and $h \mid 2 g$. It is also clear that for each $u \in D$ the $\mathbb{Q}_{2}$-dimension of $u(V)$ is divisible by $h$.

Second, we claim that $h$ is greater than 1 . Indeed, if $h=1$ then $G \subset \operatorname{End}_{D}(V)=$ $D$; in particular, $G$ is commutative, which could not be the case since $G$ maps surjectively onto noncommutative $\mathbf{A}_{2 g}$. 
Now, assume that $D$ is a field. If $e=1$ then $D=\mathbb{Q}_{2}$ and we are done. So further we assume that $e>1$. Then $V$ carries the natural structure of a $D$-vector space and $G \subset \operatorname{Aut}_{D}(V)$. Clearly,

$$
\operatorname{dim}_{D}(V)=\frac{1}{e} \operatorname{dim}_{\mathbb{Q}_{2}}(V)=\frac{2 g}{e} \leq \frac{2 g}{2}=g<2 g-2 .
$$

Corollary 2.5 applied to $m=2 g$ and $L=D$ tells us that it could not be the case.

Now assume that $D$ is not a field, i.e., it splits into a direct sum $D=D_{1} \oplus D_{2}$ of two non-zero commutative semisimple $\mathbb{Q}_{2}$-algebras. Let $e_{i}$ be the identity element of $D_{i}$ for $i=1,2$. Clearly, both $e_{i}$ 's viewed as elements of $\operatorname{End}_{\mathbb{Q}_{2}}(V)$ are idempotents; in addition, $e_{1} e_{2}=e_{2} e_{1}=0$. Then $V=V_{1} \oplus V_{2}$ where $V_{i}=e_{i}(V)$. Clearly, both $V_{i}$ 's are $G$-invariant; in addition $\operatorname{dim}_{\mathbb{Q}_{2}}\left(V_{i}\right)$ is divisible by $h$ for $i=1,2$. Since $h>1$ and

$$
\operatorname{dim}_{\mathbb{Q}_{2}}\left(V_{1}\right)+\operatorname{dim}_{\mathbb{Q}_{2}}\left(V_{2}\right)=\operatorname{dim}_{\mathbb{Q}_{2}}(V)=2 g
$$

we conclude that $\operatorname{dim}_{\mathbb{Q}_{2}}\left(V_{i}\right) \neq 1,2 g-1$. This contradicts to the already proven assertion (i).

\section{Abelian Varieties}

Let $F$ be a field, $\bar{F}$ its algebraic closure and $\operatorname{Gal}(F):=\operatorname{Aut}(\bar{F} / F)$ the absolute Galois group of $F$.

Lemma 3.1. Let $F_{1} / F$ and $F_{2} / F$ be two finite Galois extensions of fields. Suppose that $G_{1}=\operatorname{Gal}\left(F_{1} / F\right)$ is a solvable group and $G_{2}=\operatorname{Gal}\left(F_{2} / F\right)$ is simple nonabelian. Then $F_{1}$ and $F_{2}$ are linearly disjoint over $F$. In particular, the composition

$$
\operatorname{Gal}\left(F_{1} F_{2} / F_{1}\right) \subset \operatorname{Gal}\left(F_{1} F_{2} / F\right) \rightarrow \operatorname{Gal}\left(F_{2} / F\right)
$$

is a group isomorphism $\operatorname{Gal}\left(F_{1} F_{2} / F_{1}\right) \cong \operatorname{Gal}\left(F_{2} / F\right)=G_{2}$. Here $F_{1} F_{2}$ is the compositum of $F_{1}$ and $F_{2}$.

Proof. The groups $G_{1}$ and $G_{2}$ have no isomorphic quotient except the trivial one. It follows from Goursat's Lemma [43, Definition 4.1 and Remark 4.4(ii)] that every subgroup $G$ of $G_{1} \times G_{2}$ that maps surjectively on both factors $G_{1}$ and $G_{2}$ must coincide with $G_{1} \times G_{2}$. In order to finish the proof, one has to apply this observation to

$$
G=\operatorname{Gal}\left(F_{2} F_{2} / F\right) \subset \operatorname{Gal}\left(F_{1} / F\right) \times \operatorname{Gal}\left(F_{2} / F\right)=G_{1} \times G_{2} .
$$

If $X$ is an abelian variety of positive dimension over $\bar{F}$ then we write $\operatorname{End}(X)$ for the ring of all its $\bar{F}$-endomorphisms and $\operatorname{End}^{0}(X)$ for the corresponding $\mathbb{Q}$ algebra $\operatorname{End}(X) \otimes \mathbb{Q}$. We write $\operatorname{End}_{F}(X)$ for the ring of all $F$-endomorphisms of $X$ and $\operatorname{End}_{F}^{0}(X)$ for the corresponding $\mathbb{Q}$-algebra $\operatorname{End}_{F}(X) \otimes \mathbb{Q}$ and $\mathrm{C}$ for the center of $\operatorname{End}^{0}(X)$. Both $\operatorname{End}^{0}(X)$ and $\operatorname{End}_{F}^{0}(X)$ are semisimple finite-dimensional $\mathbb{Q}$-algebras.

The group $\operatorname{Gal}(F)$ of $F$ acts on $\operatorname{End}(X)$ (and therefore on $\operatorname{End}^{0}(X)$ ) by ring (resp. algebra) automorphisms and

$$
\operatorname{End}_{F}(X)=\operatorname{End}(X)^{\operatorname{Gal}(F)}, \operatorname{End}_{F}^{0}(X)=\operatorname{End}^{0}(X)^{\operatorname{Gal}(F)},
$$

since every endomorphism of $X$ is defined over a finite separable extension of $F$.

If $n$ is a positive integer that is not divisible by $\operatorname{char}(F)$ then we write $X_{n}$ for the kernel of multiplication by $n$ in $X(\bar{F})$; the commutative group $X_{n}$ is a free 
$\mathbb{Z} / n \mathbb{Z}$-module of rank $2 \operatorname{dim}(X)$ [20, End of Sect. 6, p. 64]. In particular, if $n=2$ then $X_{2}$ is an $\mathbb{F}_{2}$-vector space of dimension $2 \operatorname{dim}(X)$.

If $X$ is defined over $F$ then $X_{n}$ is a Galois submodule in $X(\bar{F})$ and all points of $X_{n}$ are defined over a finite separable extension of $F$. We write $\bar{\rho}_{n, X, F}: \operatorname{Gal}(F) \rightarrow$ $\operatorname{Aut}_{\mathbb{Z} / n \mathbb{Z}}\left(X_{n}\right)$ for the corresponding homomorphism defining the structure of the Galois module on $X_{n}$,

$$
\tilde{G}_{n, X, F} \subset \operatorname{Aut}_{\mathbb{Z} / n \mathbb{Z}}\left(X_{n}\right)
$$

for its image $\bar{\rho}_{n, X, F}(\operatorname{Gal}(F))$ and $F\left(X_{n}\right)$ for the field of definition of all points of $X_{n}$. Clearly, $F\left(X_{n}\right)$ is a finite Galois extension of $F$ with Galois group $\operatorname{Gal}\left(F\left(X_{n}\right) / F\right)=$ $\tilde{G}_{n, X, F}$. If $n=2$ then we get a natural faithful linear representation

$$
\tilde{G}_{2, X, F} \subset \operatorname{Aut}_{\mathbb{F}_{2}}\left(X_{2}\right)
$$

of $\tilde{G}_{2, X, F}$ in the $\mathbb{F}_{2}$-vector space $X_{2}$.

If $F_{1} / F$ is a finite algebraic extension then $F_{1}\left(X_{n}\right)$ coincides with the compositum $F_{1} F\left(X_{n}\right)$ of $F_{1}$ and $F\left(X_{n}\right)$

Lemma 3.2. Let $F_{1} / F$ be a finite solvable Galois extension of fields. If $\tilde{G}_{n, X, F}$ is a simple nonabelian group then $F_{1}$ and $F\left(X_{n}\right)$ are linearly disjoint over $F$ and $\tilde{G}_{n, X, F_{1}}=\tilde{G}_{n, X, F}$.

Proof. The result follows from Lemma 3.1 combined with the equality $F_{1}\left(X_{n}\right)=$ $F_{1} F\left(X_{n}\right)$.

Now and until the end of this Section we assume that $\operatorname{char}(F) \neq 2$. It is known [26] that all endomorphisms of $X$ are defined over $F\left(X_{4}\right)$; this gives rise to the natural homomorphism

$$
\kappa_{X, 4}: \tilde{G}_{4, X, F} \rightarrow \operatorname{Aut}\left(\operatorname{End}^{0}(X)\right)
$$

and $\operatorname{End}_{F}^{0}(X)$ coincides with the subalgebra $\operatorname{End}^{0}(X)^{\tilde{G}_{4, X, F}}$ of $\tilde{G}_{4, X, F}$-invariants [45, Sect. 1].

The field inclusion $F\left(X_{2}\right) \subset F\left(X_{4}\right)$ induces a natural surjection [45, Sect. 1]

$$
\tau_{2, X}: \tilde{G}_{4, X, F} \rightarrow \tilde{G}_{2, X, F} .
$$

Definition 3.3. We say that $F$ is 2-balanced with respect to $X$ if $\tau_{2, X}$ is a minimal cover. (See [8].)

Remark 3.4. Clearly, there always exists a subgroup $H \subset \tilde{G}_{4, X, F}$ such that the induced homomorphism $H \rightarrow \tilde{G}_{2, X, F}$ is surjective and a minimal cover. Let us put $L=F\left(X_{4}\right)^{H}$. Clearly,

$$
F \subset L \subset F\left(X_{4}\right), L \bigcap F\left(X_{2}\right)=F
$$

and $L$ is a maximal overfield of $F$ that enjoys these properties. It is also clear that $H$ and $L$ can be chosen that

$$
\begin{gathered}
F \subset L \subset F\left(X_{4}\right), L \bigcap F\left(X_{2}\right)=F, \\
F\left(X_{2}\right) \subset L\left(X_{2}\right), L\left(X_{4}\right)=F\left(X_{4}\right), \tilde{G}_{2, X, L}=\tilde{G}_{2, X, F}
\end{gathered}
$$

and $L$ is 2-balanced with respect to $X$ (see [8, Remark 2.3]).

We will need the following three results from previous work. 
Theorem 3.5. Suppose that $E:=\operatorname{End}_{F}^{0}(X)$ is a field that contains the center $\mathrm{C}$ of $\operatorname{End}^{0}(X)$. Let $\mathrm{C}_{X, F}$ be the centralizer of $\operatorname{End}_{F}^{0}(X)$ in $\operatorname{End}^{0}(X)$.

Then:

(i) $\mathrm{C}_{X, F}$ is a central simple E-subalgebra in $\operatorname{End}^{0}(X)$. In addition, the centralizer of $\mathrm{C}_{X, F}$ in $\operatorname{End}^{0}(X)$ coincides with $E=\operatorname{End}_{F}^{0}(X)$ and

$$
\operatorname{dim}_{E}\left(\mathrm{C}_{X, F}\right)=\frac{\operatorname{dim}_{\mathrm{C}}\left(\operatorname{End}^{0}(X)\right)}{[E: \mathrm{C}]^{2}} .
$$

(ii) Assume that $F$ is 2-balanced with respect to $X$ and $\tilde{G}_{2, X, F}$ is a non-abelian simple group. If $\operatorname{End}^{0}(X) \neq E$ (i.e., not all endomorphisms of $X$ are defined over $F$ ) then there exist a finite perfect group $\Pi \subset \mathrm{C}_{X, F}^{*}$ and a surjective homomorphism $\Pi \rightarrow \tilde{G}_{2, X, F}$ that is a minimal cover.

Proof. This is Theorem 2.4 of [8].

Lemma 3.6. Assume that $X_{2}$ does not contain proper non-trivial $\tilde{G}_{2, X, F}$-invariant even-dimensional subspaces and the centralizer $\operatorname{End}_{\tilde{G}_{2, X, F}}\left(X_{2}\right)$ has $\mathbb{F}_{2}$-dimension 2.

Then $X$ is $F$-simple and $\operatorname{End}_{F}^{0}(X)$ is either $\mathbb{Q}$ or a quadratic field.

Proof. This is Lemma 3.4 of [46].

Lemma 3.7. Let us assume that $g:=\operatorname{dim}(X)>0$ and the center of $\operatorname{End}^{0}(X)$ is a field, i.e, $\operatorname{End}^{0}(X)$ is a simple $\mathbb{Q}$-algebra.

Then:

(i) $\operatorname{dim}_{\mathbb{Q}}\left(\operatorname{End}^{0}(X)\right)$ divides $(2 g)^{2}$.

(ii) If $\operatorname{dim}_{\mathbb{Q}}\left(\operatorname{End}^{0}(X)\right)=(2 g)^{2}$ then $\operatorname{char}(F)>0$ and $X$ is a supersingular abelian variety.

Proof. This is Lemma $3.5^{1}$ of [46].

Theorem 3.8. Let $g \geq 4$ be an integer and $B$ a $2 g$-element set. Let $X$ be a $g$ dimensional abelian variety over $F$. Suppose that there exists a group isomorphism $\tilde{G}_{2, X, F} \cong \operatorname{Alt}(B)$ such that the $\operatorname{Alt}(B)$-module $X_{2}$ is isomorphic to $\mathbb{F}_{2}^{B}$.

Then one of the following two conditions holds:

(i) $\operatorname{End}^{0}(X)$ is either $\mathbb{Q}$ or a quadratic field. In particular, $X$ is absolutely simple. In addition, every finite subgroup of $\operatorname{Aut}(X)$ is cyclic.

(ii) $\operatorname{char}(F)>0$ and $X$ is a supersingular abelian variety.

Proof of Theorem 3.8. By Remark 3.4, we may and will assume that $F$ is 2-balanced with respect to $X$, i.e., $\tau_{2, X}: \tilde{G}_{4, X, F} \rightarrow \tilde{G}_{2, X, F}=\mathbf{A}_{2 g}$ is a minimal cover. In particular, $\tilde{G}_{4, X, F}$ is perfect, since $\mathbf{A}_{2 g}$ is perfect. Since $\mathbf{A}_{2 g}$ does not contain a proper subgroup of index $<2 g$, it follows from Lemma 2.3(i) that $\tilde{G}_{4, X, F}$ does not contain a proper subgroup of index $<2 g$. Now Lemmas 3.6 and 2.7 imply that $\operatorname{End}_{F}^{0}(X)$ is either $\mathbb{Q}$ or a quadratic field.

Recall that $\mathrm{C}$ is the center of $\operatorname{End}^{0}(X)$.

Lemma 3.9. Either $\mathrm{C}=\mathbb{Q} \subset \operatorname{End}_{F}^{0}(X)$ or $\mathrm{C}=\operatorname{End}_{F}^{0}(X)$ is a quadratic field.

\footnotetext{
${ }^{1}$ The $Y$ in [46, Lemma 3.5] should be $X$ and the $\operatorname{End}^{0}(Y)$ should be $\operatorname{End}^{0}(X)$.
} 
Proof of Lemma 3.9. Suppose that C is not a field. Then it is a direct sum

$$
\mathrm{C}=\oplus_{i=1}^{r} \mathrm{C}_{i}
$$

of number fields $\mathrm{C}_{1}, \ldots, \mathrm{C}_{r}$ with $1<r \leq \operatorname{dim}(X)=g$. Clearly, the center $\mathrm{C}$ is a $\tilde{G}_{4, X, F}$-invariant subalgebra of $\operatorname{End}^{0}(X)$; it is also clear that $\tilde{G}_{4, X, F}$ permutes the summands $\mathrm{C}_{i}$ 's. Since $\tilde{G}_{4, X, F}$ does not contain proper subgroups of index $\leq g$, each $\mathrm{C}_{i}$ is $\tilde{G}_{4, X, F}$-invariant. This implies that the $r$-dimensional $\mathbb{Q}$-subalgebra

$$
\oplus_{i=1}^{r} \mathbb{Q} \subset \oplus_{i=1}^{r} \mathrm{C}_{i}
$$

consists of $\tilde{G}_{4, X, F}$-invariants and therefore lies in $\operatorname{End}_{F}^{0}(X)$. It follows that $\operatorname{End}_{F}^{0}(X)$ has zero-divisors, which is not the case. The obtained contradiction proves that $\mathrm{C}$ is a field.

It is known [20, Sect. 21] that $\mathrm{C}$ contains a totally real number (sub)field $\mathrm{C}_{0}$ with $\left[\mathrm{C}_{0}: \mathbb{Q}\right] \mid \operatorname{dim}(X)$ and such that either $\mathrm{C}=\mathrm{C}_{0}$ or $\mathrm{C}$ is a purely imaginary quadratic extension of $C_{0}$. Since $\operatorname{dim}(X)=g$, the degree $\left[\mathrm{C}_{0}: \mathbb{Q}\right]$ divides $g$; in particular, the order of Aut $\left(\mathrm{C}_{0}\right)$ does not exceed $g$. Clearly, $\mathrm{C}_{0}$ is $\tilde{G}_{4, X, F}$-invariant; this gives us the natural homomorphism $\tilde{G}_{4, X, F} \rightarrow \operatorname{Aut}\left(\mathrm{C}_{0}\right)$, which must be trivial, because its kernel is a (normal) subgroup of index $\leq g$ and therefore coincides with the whole $\tilde{G}_{4, X, F}$. Therefore every element of $\mathrm{C}_{0}$ is $\tilde{G}_{4, X, F}$-invariant. This implies that $\tilde{G}_{4, X, F}$ acts on $\mathrm{C}$ through a certain group homomorphism $\tilde{G}_{4, X, F} \rightarrow \operatorname{Aut}\left(\mathrm{C} / \mathrm{C}_{0}\right)$ and this homomorphism is trivial, because the order of $\operatorname{Aut}\left(\mathrm{C} / \mathrm{C}_{0}\right)$ is either 1 (if $\mathrm{C}=\mathrm{C}_{0}$ ) or 2 (if $\mathrm{C} \neq \mathrm{C}_{0}$ ). So, every element of $\mathrm{C}$ is $\tilde{G}_{4, X, F}$-invariant, i.e.,

$$
\mathrm{C} \subset \operatorname{End}^{0}(X)^{\tilde{G}_{4, X, F}}=\operatorname{End}_{F}^{0}(X) .
$$

This implies that if $\mathrm{C} \neq \mathbb{Q}$ then $\operatorname{End}_{F}^{0}(X)$ is also not $\mathbb{Q}$ and therefore is a quadratic field containing $\mathrm{C}$, which implies that $\mathrm{C}=\operatorname{End}_{F}^{0}(X)$ is also a quadratic field.

It follows that $\operatorname{End}^{0}(X)$ is a simple $\mathbb{Q}$-algebra (and a central simple C-algebra). Let us put $E:=\operatorname{End}_{F}^{0}(X)$ and denote by $\mathrm{C}_{X, F}$ the centralizer of $E$ in $\operatorname{End}^{0}(X)$. We have

$$
\mathrm{C} \subset E \subset \mathrm{C}_{X, F} \subset \operatorname{End}^{0}(X) .
$$

Combining Lemma 3.9 with Theorem 3.5 and Lemma 3.7, we obtain the following assertion.

Proposition 3.10. $\quad$ (i) $\mathrm{C}_{X, F}$ is a central simple E-subalgebra in $\operatorname{End}^{0}(X)$,

$$
\operatorname{dim}_{E}\left(\mathrm{C}_{X, F}\right)=\frac{\operatorname{dim}_{\mathrm{C}}\left(\operatorname{End}^{0}(X)\right)}{[E: \mathrm{C}]^{2}}
$$

and $\operatorname{dim}_{E}\left(\mathrm{C}_{X, F}\right)$ divides $(2 \operatorname{dim}(X))^{2}=(2 g)^{2}$.

(ii) If $\operatorname{End}^{0}(X) \neq E$ (i.e., not all endomorphisms of $X$ are defined over $F$ ) then there exist a finite perfect group $\Pi \subset \mathrm{C}_{X, F}^{*}$ and a surjective homomorphism $\pi: \Pi \rightarrow \tilde{G}_{2, X, F}$ that is a minimal cover.

End of Proof of Theorem 3.8. If $\operatorname{End}^{0}(X)=E$ then we are done. If $\operatorname{dim}_{E}\left(\mathrm{C}_{X, F}\right)=(2 g)^{2}$ then

$$
\operatorname{dim}_{\mathbb{Q}}\left(\operatorname{End}^{0}(X)\right) \geq \operatorname{dim}_{C}\left(\operatorname{End}^{0}(X)\right) \geq \operatorname{dim}_{E}\left(\mathrm{C}_{X, F}\right)=(2 g)^{2}=(2 \operatorname{dim}(X))^{2}
$$


and it follows from Lemma 3.7 that $\operatorname{dim}_{\mathbb{Q}}\left(\operatorname{End}^{0}(X)\right)=(2 \operatorname{dim}(X))^{2}, \operatorname{char}(F)>0$ and $X$ is a supersingular abelian variety. So, further we may and will assume that

$$
\operatorname{End}^{0}(X) \neq E, \operatorname{dim}_{E}\left(\mathrm{C}_{X, F}\right) \neq(2 g)^{2} .
$$

We need to arrive to a contradiction. Let $\Pi \subset \mathrm{C}_{X, F}^{*}$ be as in 3.10(ii). Since $\Pi$ is perfect, $\operatorname{dim}_{E}\left(\mathrm{C}_{X, F}\right)>1$. It follows from Proposition 3.10(i) that $\operatorname{dim}_{E}\left(\mathrm{C}_{X, F}\right)=d^{2}$ where $d$ is a positive integer such that

$$
1<d<2 g, \quad d \mid 2 g \text {. }
$$

This implies that

$$
d \leq \frac{2 g}{2}=g<2 g-2
$$

Let us fix an embedding $E \hookrightarrow \mathbb{C}$ and an isomorphism $\mathrm{C}_{X, F} \otimes_{E} \mathbb{C} \cong \mathrm{M}_{d}(\mathbb{C})$. This gives us an embedding $\Pi \hookrightarrow \operatorname{GL}(d, \mathbb{C})$. Further we will identify $\Pi$ with its image in $\operatorname{GL}(d, \mathbb{C})$. Clearly, only central elements of $\Pi$ are scalars. It follows that there is a central subgroup $\mathcal{Z}$ of $\Pi$ such that the natural homomorphism $\Pi / \mathcal{Z} \rightarrow \operatorname{PGL}(d, \mathbb{C})$ is an embedding. The simplicity of $\tilde{G}_{2, X, F}=\mathbf{A}_{2 g}$ implies that $Z$ lies in the kernel of $\Pi \rightarrow \tilde{G}_{2, X, F}=\mathbf{A}_{2 g}$ and the induced map $\Pi / Z \rightarrow \tilde{G}_{2, X, F}$ is also a minimal cover. It follows from Lemma 2.3(ii) applied to $\mathcal{G}_{1}=\Pi / \mathcal{Z}$ that $d \geq 2 g-1$. However, we have seen that $d<2 g-2$. This gives us a desired contradiction.

Recall that $\operatorname{Aut}(X) \subset \operatorname{End}^{0}(X)^{*}$. Since every finite multiplicative subgroup in a field is commutative, every finite subgroup of $\operatorname{Aut}(X)$ is cyclic if $\operatorname{End}^{0}(X)$ is a field.

\section{TATE MOdUles OF ABELIAN VARIETIES}

We keep the notation and assumptions of the previous Section. Let $\ell$ be a prime different from $\operatorname{char}(F)$. Let us consider the $\ell$-adic Tate module $T_{\ell}(X)$ of $X$ that is the projective limit of $X_{\ell^{i}}(i=1,2, \ldots)$ where the transition map $X_{\ell^{i+1}} \rightarrow X_{\ell^{i}}$ is multiplication by $\ell$ [20, Sect. 18]. The Tate module $T_{\ell}(X)$ carries the natural

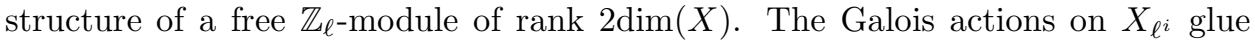
together to the continuous homomorphism

$$
\rho_{\ell, X}: \operatorname{Gal}(K) \rightarrow \operatorname{Aut}_{\mathbb{Z}_{\ell}}\left(T_{\ell}(X)\right),
$$

providing $T_{\ell}(X)$ with the structure of Galois module. The natural surjective map $T_{\ell}(X) \rightarrow X_{\ell}$ induces an isomorphism of Galois modules

$$
T_{\ell}(X) \otimes_{\mathbb{Z}_{\ell}} \mathbb{Z}_{\ell} / \ell \mathbb{Z}_{\ell}=T_{\ell}(X) / \ell T_{\ell}(X) \cong X_{\ell}
$$

We also consider the $2 \operatorname{dim}(X)$-dimensional $\mathbb{Q}_{\ell}$-vector space

$$
V_{\ell}(X):=T_{\ell}(X) \otimes_{\mathbb{Z}_{\ell}} \mathbb{Q}_{\ell} .
$$

One may view $T_{\ell}(X)$ as a $\mathbb{Z}_{\ell}$-lattice of rank $2 \operatorname{dim}(X)$ in $V_{\ell}(X)$. Let us put

$$
G_{\ell, X}=G_{\ell, X, F}:=\rho_{\ell, X}(\operatorname{Gal}(F)) \subset \operatorname{Aut}_{\mathbb{Z}_{\ell}}\left(T_{\ell}(X)\right) \subset \operatorname{Aut}_{\mathbb{Q}_{\ell}}\left(V_{\ell}(X)\right) ;
$$

it is known [30, Sect. 1.2 and Example 1.2.3] that $G_{\ell, X}$ is a compact $\ell$-adic Lie group, whose Lie algebra $\mathfrak{g}_{\ell, X}$ is a $\mathbb{Q}_{\ell}$-Lie subalgebra of $\operatorname{End}_{\mathbb{Q}_{\ell}}\left(V_{\ell}(X)\right)$.

The reduction map modulo $\ell$

$$
\text { red }: \operatorname{Aut}_{\mathbb{Z}_{\ell}}\left(T_{\ell}(X)\right) \rightarrow \operatorname{Aut}_{\mathbb{F}_{\ell}}\left(T_{\ell}(X) / \ell T_{\ell}(X)\right)=\operatorname{Aut}_{\mathbb{F}_{\ell}}\left(X_{\ell}\right)
$$

induces a continuous surjective homomorphism

$$
\pi_{\ell, X}: G_{\ell, X} \rightarrow \tilde{G}_{\ell, X, F} \subset \operatorname{Aut}_{F_{\ell}}\left(X_{\ell}\right) .
$$


Remark 4.1. Let $X$ and $Y$ be abelian varieties over $F$ and $u: X \rightarrow Y$ be an $F$-isogeny. Then $u$ induces, by functoriality, an isomorphism of Galois modules $V_{\ell}(X) \cong V_{\ell}(Y)$. This implies that there is a continuous group isomorphism between the compact profinite groups $G_{\ell, X, F}$ and $G_{\ell, Y, F}$.

Our next statement deals with the case of $\ell=2$.

Theorem 4.2. Let $g \geq 5$ be an integer and $B$ a $2 g$-element set. Let $F$ be a field of characteristic zero and $X$ a g-dimensional abelian variety over $F$. Suppose that there exists a group isomorphism $\tilde{G}_{2, X, F} \cong \operatorname{Alt}(B)$ such that the $\operatorname{Alt}(B)=\tilde{G}_{2, X, F^{-}}$ module $X_{2}$ is isomorphic to $\mathbb{F}_{2}^{B}$.

Then $\operatorname{End}(X)=\mathbb{Z}$.

Proof. By Theorem 3.8, we know that $\operatorname{End}^{0}(X)$ is either $\mathbb{Q}$ or a quadratic field. If $\operatorname{End}^{0}(X)=\mathbb{Q}$ then $\operatorname{End}(X)=\mathbb{Z}$. So, further we assume that $E:=\operatorname{End}^{0}(X)$ is a quadratic field. Clearly, $\operatorname{Aut}(E)$ is a cyclic group of order 2. Replacing if necessary, $F$ by its suitable quadratic extension and using Lemma 3.2, we may and will assume that $\operatorname{Gal}(F)$ acts trivially on $\operatorname{End}^{0}(X)$, i.e., all endomorphisms of $X$ are defined over $F$, i.e., $E=\operatorname{End}_{F}^{0}(X)$. Clearly, $E_{2}:=E \otimes_{\mathbb{Q}} \mathbb{Q}_{2}$ is a two-dimensional commutative semisimple $\mathbb{Q}_{2}$-algebra. It is well-known that there is a natural embedding

$$
E_{2} \hookrightarrow \operatorname{End}_{\operatorname{Gal}(F)} V_{2}(X) \subset \operatorname{End}_{\mathbb{Q}_{2}}\left(V_{2}(X)\right) .
$$

This implies that $E_{2}$ sits in the centralizer of $G_{2, X, F}$. It is also known that the $E_{2}$-module $V_{2}(X)$ is free [24, Theorem 2.1.1]. However, applying Theorem 2.8 to $V=V_{2}(X), T=T_{2}(X), G=G_{2, X, F}$ and $D=E_{2}$, we conclude that $E_{2}=\mathbb{Q}_{2}$, which could not be the case, since $\mathbb{Q}_{2}$ is the one-dimensional $\mathbb{Q}_{2}$-algebra.

Theorem 4.3. Suppose that $X$ is as in Theorem 3.8. Suppose that $Y$ is a $g$ dimensional abelian variety over $F$ that enjoys one of the following properties:

(i) $\tilde{G}_{2, Y, F}$ is solvable;

(ii) The fields $F\left(X_{2}\right)$ and $F\left(Y_{2}\right)$ are linearly disjoint over $F$.

If $\operatorname{char}(F)=0$ then $X$ and $Y$ are not isogenous over $\bar{F}$.

Proof of Theorem 4.3. If $\tilde{G}_{2, Y, F}$ is solvable then it follows from Lemma 3.1 that $F\left(X_{2}\right)$ and $F\left(Y_{2}\right)$ are linearly disjoint over $F$, since $\tilde{G}_{2, X, F}=\operatorname{Alt}(B) \cong \mathbf{A}_{2 g}$ is simple non-abelian. So, $F\left(X_{2}\right)$ and $F\left(Y_{2}\right)$ are linearly disjoint over $F$. Since the compositum of $F\left(X_{2}\right)$ and $F\left(Y_{2}\right)$ coincides with $F\left(Y_{2}\right)\left(X_{2}\right)$, we conclude that

$$
\tilde{G}_{2, X, F}=\tilde{G}_{2, X, F\left(Y_{2}\right)} \text {. }
$$

Replacing the ground field $F$ by $F\left(Y_{2}\right)$, we may and will assume that $\tilde{G}_{2, Y, F}=\{1\}$, i.e., the Galois module $Y_{2}$ is trivial. It follows that $G_{2, Y, F} \subset \operatorname{Id}+2 \operatorname{End}_{\mathbb{Z}_{2}}\left(T_{2}(Y)\right)$; in particular, $G_{2, Y, F}$ is a pro-2-group. By Theorem $3.8, \operatorname{End}^{0}(X)$ is either $\mathbb{Q}$ or a quadratic field say, $L$. In the former case all the endomorphisms of $X$ are defined over $F$. In the latter case, all the endomorphisms of $X$ are defined either over $F$ or over a certain quadratic extension of $F$, because the automorphism group of $L$ is the cyclic group of order 2. Replacing if necessary $F$ by the corresponding quadratic extension, we may and will assume that all the endomorphisms of $X$ are defined over $F$. In particular, all the automorphisms of $X$ are defined over $F$.

There is still a continuous surjective homomorphism

$$
G_{2, X, F} \rightarrow \tilde{G}_{2, X, F}=\operatorname{Alt}(B) \cong \mathbf{A}_{2 g}
$$


and therefore $G_{2, X, F}$ is not a pro-2-group. This implies that there does not exist a continuous isomorphism between $G_{2, X, F}$ and $G_{2, Y, F}$. It follows from Remark 4.1 that $X$ and $Y$ are not isogenous over $F$.

Let $u: X \rightarrow Y$ be an $\bar{F}$-isogeny of abelian varieties. As we have seen, $u$ could not be defined over $F$. However, there exists a finite Galois extension $F_{u} / F$ such that $u$ is defined over $F_{u}$.

Let us consider the 1-cocycle

$$
c: \operatorname{Gal}\left(F_{u} / F\right) \rightarrow \operatorname{End}^{0}(X)^{*}, \sigma \mapsto c_{\sigma}:=u^{-1} \sigma(u) .
$$

Since the Galois group acts trivially on $\operatorname{End}^{0}(X)$, the map $c: \operatorname{Gal}\left(F_{u} / F\right) \rightarrow$ $\operatorname{End}^{0}(X)^{*}$ is a group homomorphism, whose image is a finite subgroup of $\operatorname{End}^{0}(X)^{*}$ and therefore is cyclic, thanks to Theorem 3.8(i). Therefore there is a finite cyclic subextension $F^{\prime} / F$ such that

$$
c_{\sigma}=1 \forall \sigma \in \operatorname{Gal}\left(F_{u} / F^{\prime}\right) \subset \operatorname{Gal}\left(F_{u} / F\right) .
$$

It follows that $u$ is defined over $F^{\prime}$ and therefore the $\operatorname{Gal}\left(F^{\prime}\right)$-modules $V_{2}(X)$ and $V_{2}(Y)$ are isomorphic. In particular, there is a continuous group isomorphism between $G_{2, X, F^{\prime}}$ and $G_{2, Y, F^{\prime}}$. But $G_{2, X, F^{\prime}} \subset G_{2, X, F}$ is still a pro-2-group. On the other hand, since $F^{\prime} / F$ is cyclic and $\operatorname{Gal}\left(F\left(X_{2}\right) / F\right)=\tilde{G}_{2, X, F} \cong \mathbf{A}_{2 g}$ is simple non-abelian, Lemma 3.2 tells us that

$$
\tilde{G}_{2, X, F^{\prime}}=\tilde{G}_{2, X, F} \cong \mathbf{A}_{2 g}
$$

and there is a surjective continuous homomorphism

$$
G_{2, X, F^{\prime}} \rightarrow \tilde{G}_{2, X, F^{\prime}} \cong \mathbf{A}_{2 g} .
$$

In particular, $G_{2, X, F^{\prime}}$ is not a pro-2-group. there does not exist a continuous group isomorphism

\section{Points OF ORder 2}

5.1. Let $K$ be a field of characteristic different from 2 , let $f(x) \in K[x]$ be a polynomial of odd degree $n \geq 5$ and without multiple roots. Let $C_{f}$ be the hyperelliptic curve $y^{2}=f(x)$ and $J\left(C_{f}\right)$ the jacobian of $C_{f}$. The Galois module $J\left(C_{f}\right)_{2}$ of points of order 2 admits the following description.

Let $\mathbb{F}_{2}^{\mathfrak{R}_{f}}$ be the $n$-dimensional $\mathbb{F}_{2}$-vector space of functions $\varphi: \mathfrak{R}_{f} \rightarrow \mathbb{F}_{2}$ provided with the natural structure of $\operatorname{Gal}(f) \subset \operatorname{Perm}\left(\Re_{f}\right)$-module. The canonical surjection

$$
\operatorname{Gal}(K) \rightarrow \operatorname{Gal}\left(K\left(\Re_{f}\right) / K\right)=\operatorname{Gal}(f)
$$

provides $\mathbb{F}_{2}^{\Re_{f}}$ with the structure of $\operatorname{Gal}(K)$-module. Let us consider the hyperplane

$$
\left(\mathbb{F}_{2}^{\Re_{f}}\right)^{0}:=\left\{\varphi: \mathfrak{R}_{f} \rightarrow \mathbb{F}_{2} \mid \sum_{\alpha \in \mathfrak{R}_{f}} \varphi(\alpha)=0\right\} \subset \mathbb{F}_{2}^{\mathfrak{R}_{f}} .
$$

Clearly, $\left(\mathbb{F}_{2}^{\mathfrak{R}_{f}}\right)^{0}$ is a Galois submodule in $\mathbb{F}_{2}^{\mathfrak{R}_{f}}$.

It is well-known (see, for instance, [41]) that if $n$ is odd then the Galois modules $J\left(C_{f}\right)_{2}$ and $\left(\mathbb{F}_{2}^{\mathfrak{R}_{f}}\right)^{0}$ are isomorphic. It follows that if $X=J\left(C_{f}\right)$ then $\tilde{G}_{2, X, K}=$ $\operatorname{Gal}(f)$ and $K\left(J\left(C_{f}\right)_{2}\right)=K\left(\Re_{f}\right)$.

Lemma 5.2. Suppose that $n=\operatorname{deg}(f)$ is odd and $f(x)=(x-t) h(x)$ with $t \in K$ and $h(x) \in K[x]$. Then $\tilde{G}_{2, J\left(C_{f}\right), K} \cong \operatorname{Gal}(h)$ and the Galois modules $J\left(C_{f}\right)_{2}$ and $\mathbb{F}_{2}^{\mathfrak{R}_{h}}$ are isomorphic. 
Proof. We have

$$
\tilde{G}_{2, X, K}=\operatorname{Gal}(f)=\operatorname{Gal}(h) .
$$

In order to prove the second assertion, it suffices to check that the Galois modules $\left(\mathbb{F}_{2}^{\Re_{f}}\right)^{0}$ and $\mathbb{F}_{2}^{\Re_{h}}$ are isomorphic. Recall that $\mathfrak{R}_{f}$ is the disjoint union of $\mathfrak{R}_{h}$ and $\{t\}$. Consider the map $\left(\mathbb{F}_{2}^{\Re_{f}}\right)^{0} \rightarrow \mathbb{F}_{2}^{\Re_{h}}$ that sends the function $\varphi: \mathfrak{R}_{f} \rightarrow \mathbb{F}_{2}$ to its restriction to $\mathfrak{R}_{h}$. Obviously, this map is an isomorphism of Galois modules.

Corollary 5.3. Suppose that $\operatorname{char}(K)=0, n=\operatorname{deg}(f)=2 g+1$ is odd and $f(x)=$ $(x-t) h(x)$ with $t \in K$ and $h(x) \in K[x]$. Assume also that $\operatorname{Gal}(h)=\operatorname{Alt}\left(\Re_{h}\right) \cong \mathbf{A}_{2 g}$.

(i) If $g \geq 4$ then $\operatorname{End}^{0}\left(J\left(C_{f}\right)\right)$ is either $\mathbb{Q}$ or a quadratic field.

(i) If $g \geq 5$ then $\operatorname{End}\left(J\left(C_{f}\right)\right)=\mathbb{Z}$.

Proof of Corollary 5.3. Let us put $K=F, X=J\left(C_{f}\right)$ and $B=\mathfrak{R}_{h}$. Then assertion (i) is an immediate corollary of Lemma 5.2 and Theorem 3.8. The assertion (ii) follows from Theorem 4.2.

Proof of Theorem 1.4 and Theorem 1.5. Replacing if necessary, $K$ by its suitable quadratic extension, we may and will assume that $\operatorname{Gal}(h)=\mathbf{A}_{2 g}$ (recall that $n=$ $2 g+1)$. Now Theorem 1.4 is an immediate corollary of Corollary 5.3(i). Theorem 1.5 follows from Corollary 5.3(ii).

Theorem 5.4. Suppose that $\operatorname{char}(K)=0, n=\operatorname{deg}(f) \geq 9$ is odd and $f(x)=$ $(x-t) h(x)$ with $t \in K$ and $h(x) \in K[x]$. Assume also that $\operatorname{Gal}(h)$ is either $\mathbf{S}_{n-1}$ or $\mathbf{A}_{n-1}$. Suppose that $f_{1}(x) \in K[x]$ is a degree $n$ polynomial without multiple roots that enjoys one of the following properties:

(i) $f_{1}(x)$ splits into a product of linear factors over $K$.

(ii) $f_{1}(x)=\left(x-t_{1}\right) h_{1}(x)$ with $t_{1} \in K$ and $h_{1}(x) \in K[x]$. In addition, the splitting fields of $h(x)$ and $h_{1}(x)$ are linearly disjoint over $K$.

(iii) The splitting fields of $h(x)$ and $f_{1}(x)$ are linearly disjoint over $K$.

Then the jacobians $J\left(C_{f}\right)$ and $J\left(C_{f_{1}}\right)$ are not isogenous over $\bar{K}$.

Proof. It suffices to do the case when the splitting fields of $f(x)$ and $f_{1}(x)$ are linearly disjoint over $K$. (This condition is obviously fulfilled in the cases (i) and (ii).) Let us put $X=J\left(C_{f}\right), Y=J\left(C_{f_{1}}\right)$. According to Sect. 5.1,

$$
K\left(J\left(C_{f}\right)_{2}\right)=K\left(\Re_{f}\right), K\left(J\left(C_{f_{1}}\right)_{2}\right)=K\left(\Re_{f_{1}}\right) .
$$

Now the result follows from Theorem 4.3 combined with Lemma 5.2.

Theorem 5.5. Suppose that $\operatorname{char}(K)=0, n=2 g+2=\operatorname{deg}(f) \geq 10$ is even and $f(x)=\left(x-t_{1}\right)\left(x-t_{2}\right) u(x)$ with

$$
t_{1}, t_{2} \in K, t_{1} \neq t_{2}, u(x) \in K[x], \operatorname{deg}(u)=n-2 .
$$

If $\operatorname{Gal}(u)=\mathbf{S}_{n-2}$ or $\mathbf{A}_{n-2}$ then $\operatorname{End}^{0}\left(J\left(C_{f}\right)\right)$ is either $\mathbb{Q}$ or a quadratic field; in particular, $J\left(C_{f}\right)$ is an absolutely simple abelian variety. In addition, if $n \geq 12$ then $\operatorname{End}\left(J\left(C_{f}\right)\right)=\mathbb{Z}$.

Proof. Let us put $h(x)=\left(x-t_{2}\right) u(x)$. We have $f(x)=\left(x-t_{1}\right) h(x)$. As in the proof of Theorem 1.3, let us consider the degree $(n-1)$ polynomials

$$
h_{1}(x)=h\left(x+t_{1}\right)=\left(x+t_{1}-t_{2}\right) u\left(x+t_{1}\right), h_{2}(x)=x^{n-1} h_{1}(1 / x) \in K[x] .
$$


We have

$$
\begin{gathered}
\mathfrak{R}_{h_{1}}=\left\{\alpha-t_{1} \mid \alpha \in \mathfrak{R}_{h}\right\}=\left\{\alpha-t_{1}+t_{2} \mid \alpha \in \mathfrak{R}_{u}\right\} \bigcup\left\{t_{2}-t_{1}\right\}, \\
\mathfrak{R}_{h_{2}}=\left\{\frac{1}{\alpha-t_{1}} \mid \alpha \in \mathfrak{R}_{u}\right\} \bigcup\left\{\frac{1}{t_{2}-t_{1}}\right\} .
\end{gathered}
$$

This implies that

$$
K\left(\Re_{h_{2}}\right)=K\left(\Re_{h_{1}}\right)=K\left(\Re_{u}\right)
$$

and

$$
h_{2}(x)=\left(x-\frac{1}{t_{2}-t_{1}}\right) v(x)
$$

where $v(x) \in K[x]$ is a degree $(n-2)$ polynomial with $K\left(\Re_{v}\right)=K\left(\Re_{u}\right)$; in particular, $\operatorname{Gal}(v)=\operatorname{Gal}(u)=\mathbf{S}_{n-2}$ or $\mathbf{A}_{n-2}$. Again, the standard substitution

$$
x_{1}=1 /\left(x-t_{1}\right), y_{1}=y /\left(x-t_{1}\right)^{g+1}
$$

establishes a birational $K$-isomorphism between $C_{f}$ and a hyperelliptic curve

$$
C_{h_{2}}: y_{1}^{2}=h_{2}\left(x_{1}\right) \text {. }
$$

Now the result follows from Theorems 1.4 and 1.5 applied to $h_{2}\left(x_{1}\right)$.

\section{FAMilies of hyperelliptic CURVES}

Throughout this Section, $K$ is a field of characteristic different from $2, \bar{K}$ its algebraic closure and $\operatorname{Gal}(K)=\operatorname{Aut}(\bar{K} / K)$ its absolute Galois group. Let $n \geq 5$ be an integer, $f(x) \in K[x]$ a degree $n$ polynomial without multiple roots, $\Re_{f} \subset \bar{K}$ the $n$-element set of its roots, $K\left(\Re_{f}\right) \subset \bar{K}$ the splitting field of $f(x)$ and $\mathrm{Gal}(f)=$ $\operatorname{Gal}\left(K\left(\Re_{f}\right) / K\right)$ the Galois group of $f(x)$ over $K$. One may view $\operatorname{Gal}(f)$ as a certain group of permutations of $\mathfrak{R}_{f}$. Let $C_{f}: y^{2}=f(x)$ the corresponding hyperelliptic curve of genus $\lfloor(n-1) / 2\rfloor$.

Theorem 6.1. Let $n \geq 7$ be an integer, $h(x) \in K[x]$ an irreducible polynomial of degree $n-1$, whose Galois group is either $\mathbf{S}_{n-1}$ or $\mathbf{A}_{n-1}$. For every $t \in K$ let $f_{t}(x)=(x-t) h(x)$ and $D(t)=C_{f_{t}}$. Then there exists a finite set $B=B(h)$ that enjoys the following properties.

If $t_{1}$ and $t_{2}$ are distinct elements of $K$ such that the hyperelliptic curves $D\left(t_{1}\right)$ and $D\left(t_{2}\right)$ are isomorphic over $\bar{K}$ then both $t_{1}$ and $t_{2}$ belong to $B$.

Remark 6.2. It is well known ([12, Ch. 2, Sect. 3, pp. 253-255], [6, Ch. VIII, Sect. 3]) that the hyperelliptic curves $D\left(t_{1}\right)$ and $D\left(t_{2}\right)$ are isomorphic over $\bar{K}$ if and only if there exists a fractional linear transformation $T \in \mathrm{PGL}_{2}(\bar{K})=\operatorname{Aut}\left(\mathbb{P}^{1}\right)$ that sends the branch points of the canonical double cover $D\left(t_{1}\right) \rightarrow \mathbb{P}^{1}$ to the branch points of the canonical double cover $D\left(t_{2}\right) \rightarrow \mathbb{P}^{1}$.

Proof of Theorem 6.1. Let $\mathfrak{R}_{h} \subset \bar{K}$ be the $(n-1)$-element set of roots of $h(x)$. Let us consider two distinct ordered triples

$$
\left\{\alpha_{1}, \alpha_{2}, \alpha_{3}\right\},\left\{\beta_{1}, \beta_{2}, \beta_{3}\right\} \subset \mathfrak{R}_{h} \subset \bar{K} \subset \mathbb{P}^{1}(\bar{K})
$$

of roots of $h(x)$. There exists exactly one fractional-linear transformation

$$
T=T\left(\alpha_{1}, \alpha_{2}, \alpha_{3} ; \beta_{1}, \beta_{2}, \beta_{3}\right) \in \mathrm{PGL}_{2}(\bar{K})
$$

such that

$$
T\left(\alpha_{1}\right)=\beta_{1}, T\left(\alpha_{2}\right)=\beta_{2}, T\left(\alpha_{3}\right)=\beta_{3} .
$$


We write $J_{1}=J_{1}(h)$ for the set of all those T's (for all choices of $\alpha$ 's and $\beta$ 's). Clearly, $J_{1}$ is a finite subset in $\mathrm{PGL}_{2}(\bar{K})$, whose cardinality bounded by a constant depending only on $n$. It is also clear that $J_{1}$ does not contain the identity element and

$$
J_{1}=\left\{T^{-1} \mid T \in J_{1}\right\} .
$$

In addition, $J_{1}$ is $\operatorname{Gal}(K)$-stable.

Lemma 6.3. Suppose that $\mathrm{Gal}(h)$ is either $\mathbf{S}_{n-1}$ or $\mathbf{A}_{n-1}$. Then the only fractionallinear transformation $U \in \mathrm{PGL}_{2}(\bar{K})$ that sends $\mathfrak{R}_{h}$ into itself is the identity map.

Proof of Lemma 6.3. Replacing if necessary $K$ by its suitable quadratic extension, we may and will assume that $\operatorname{Gal}(h)=\mathbf{A}_{n-1}$. Let us consider the subgroup

$$
G=\left\{U \in \mathrm{PGL}_{2}(\bar{K}) \mid U\left(\mathfrak{R}_{h}\right)=\mathfrak{R}_{h}\right\} \subset \mathrm{PGL}_{2}(\bar{K}) .
$$

Since $\#\left(\mathfrak{R}_{h}\right)=n-1 \geq 6>3, G$ is a finite group and the natural homomorphism $G \rightarrow \operatorname{Perm}\left(\Re_{h}\right)=\mathbf{S}_{n-1}$ is injective; we write

$$
G_{0} \subset \operatorname{Perm}\left(\Re_{h}\right)=\mathbf{S}_{n-1}
$$

for its image. Clearly, $G_{0} \cong G$. Since $\mathfrak{R}_{h}$ is Galois-invariant, $G_{0}$ is stable with respect to the conjugation by elements of $\operatorname{Gal}(K)$. Since the image of $\operatorname{Gal}(K)$ in $\operatorname{Perm}\left(\Re_{h}\right)$ is $\mathbf{A}_{n-1}$, the subgroup $G_{0} \subset \mathbf{S}_{n-1}$ is stable with respect to conjugation by elements of $\mathbf{A}_{n-1}$. Since $n-1>5$, it follows that $G_{0}$ is either trivial or $\mathbf{S}_{n-1}$ or $\mathbf{A}_{n-1}$. Therefore $G$ is either trivial or isomorphic to $\mathbf{S}_{n-1}$ or $\mathbf{A}_{n-1}$. Now the classifications of finite subgroups of $\mathrm{PSL}_{2}(\bar{K})=\mathrm{PGL}_{2}(\bar{K})$ tells us that $G$ is trivial and we are done.

Proposition 6.4. Suppose that $\mathrm{Gal}(h)$ is either $\mathbf{S}_{n-1}$ or $\mathbf{A}_{n-1}$. If $T \in J_{1}$ then there exists $\sigma \in \operatorname{Gal}(K)$ such that $\sigma(T) \neq T$.

Proof of Proposition 6.4. Assume that $\sigma(T)=T$ for all $\sigma \in \operatorname{Gal}(K)$. Since $T \in J_{1}$, there exists $\alpha \in \mathfrak{R}_{h}$ such that $T(\alpha) \in \mathfrak{R}_{h}$. Since $\mathfrak{R}_{h}$ coincides with the Galois orbit of $\alpha$, we have $T\left(\mathfrak{R}_{h}\right)=\mathfrak{R}_{h}$. By Lemma $6.3, T$ is the identity map. But $J_{1}$ does not contain the identity element. The obtained contradiction proves the desired result.

\section{Continuing the proof of Theorem 6.1.}

Let $B_{1}$ be the set of all $t \in K$ such that there exist $\alpha \in \Re_{h}$ and $T \in J_{1}$ such that $t=T(\alpha)$. Let $B_{2}$ be the set of all $t \in K$ such that there exist $\sigma \in \operatorname{Gal}(K)$ and $T \in J_{1}$ such that $\sigma(T) \neq T$ and $t$ is a fixed point of $\sigma(T) T^{-1}$. Let $B_{3}$ be the set of all $t \in K$ such that there exists $T \in J_{1}$ such that $t=T(\infty)$.

Clearly, $B_{1}, B_{2}$ and $B_{3}$ are finite sets, whose cardinalities are bounded by a constant depending only on $n$. Let us put

$$
B=B(h)=B_{1} \cup B_{2} \cup B_{3} .
$$

End of the proof of Theorem 6.1. First assume that $n$ is even. Then the set of the ramification points of the double cover

$$
D(t) \rightarrow \mathbb{P}^{1},(x, y) \mapsto x
$$

coincides with $\mathfrak{R}_{f_{t}}=\{t\} \cup \Re_{h}$. So, if $t_{1} \neq t_{2}$ and $D\left(t_{1}\right)$ and $D\left(t_{2}\right)$ are isomorphic over $\bar{K}$ then there exists $T \in \mathrm{PGL}_{2}(\bar{K})$ such that

$$
T\left(\left\{t_{1}\right\} \cup \mathfrak{R}_{h}\right)=\left\{t_{2}\right\} \cup \mathfrak{R}_{h} .
$$


By Lemma $6.3, T\left(\mathfrak{R}_{h}\right) \neq \mathfrak{R}_{h}$. This implies that there is $\beta \in \mathfrak{R}_{h}$ with $T(\beta)=t_{2}$. It follows that $T\left(\mathfrak{R}_{h} \backslash\{\beta\}\right) \subset \mathfrak{R}_{h}$. Since $\#\left(\mathfrak{R}_{h} \backslash\{\beta\}\right)=n-2>3$, the transformation $T$ lies in $J_{1}$ and therefore $t_{2} \in B_{1}$. By symmetry, $t_{1}$ also lies in $B_{1}$.

Now assume that $n$ is odd. Then the set of the ramification points of the double cover

$$
D(t) \rightarrow \mathbb{P}^{1},(x, y) \mapsto x
$$

coincides with $\{\infty\} \cup \mathfrak{R}_{f_{t}}=\{\infty\} \cup\{t\} \cup \mathfrak{R}_{h}$. So, if $t_{1} \neq t_{2}$ and $D\left(t_{1}\right)$ and $D\left(t_{2}\right)$ are isomorphic over $\bar{K}$ then there exists $T \in \mathrm{PGL}_{2}(\bar{K})$ such that

$$
T\left(\{\infty\} \cup\left\{t_{1}\right\} \cup \Re_{h}\right)=\{\infty\} \cup\left\{t_{2}\right\} \cup \Re_{h} .
$$

By Lemma 6.3, $T\left(\mathfrak{R}_{h}\right) \neq \mathfrak{R}_{h}$. So, either there is $\beta \in \mathfrak{R}_{h}$ such that $T(\beta)=t_{2}$ or $T\left(\Re_{h}\right)$ does not contain $t_{2}$ but contains $\infty$. In the former case, arguments as above prove that $t_{2} \in B_{1}$. In the latter case, either $T\left(t_{1}\right)=t_{2}$ or $T(\infty)=t_{2}$. In the former case, $\sigma(T)\left(t_{1}\right)=t_{2}$ for all $\sigma \in \operatorname{Gal}(K)$ and therefore $t_{2}$ is a fixed point of $\sigma(T) T^{-1}$, which implies that $t_{2} \in B_{2}$. In the latter case, $T(\infty)=t_{2}$ and therefore $t_{2} \in B_{3}$. However, we always have

$$
t_{2} \in B_{1} \cup B_{2} \cup B_{3}=B .
$$

By symmetry, $t_{1} \in B$.

Theorem 6.5. Suppose that $n \geq 8$ is an integer that does not equal 9. Let $K$ be a field of characteristic zero and $h(x) \in K[x]$ an irreducible polynomial of degree $n-1$. Assume also that $\operatorname{Gal}(h)$ is either $\mathbf{S}_{n-1}$ or $\mathbf{A}_{n-1}$. Then there exists a finite set $B=B(h) \subset K$ that enjoys the following properties.

Let $t_{1}$ and $t_{2}$ be distinct elements of $K$ and let $f_{1}(x)=\left(x-t_{1}\right) h(x), f_{2}(x)=$ $\left(x-t_{2}\right) h(x)$. If the abelian varieties $J\left(C_{f_{1}}\right)$ and $J\left(C_{f_{2}}\right)$ are isomorphic over $\bar{K}$ then both $t_{1}$ and $t_{2}$ belong to $B$.

Proof. Let $B(h)$ be as in Theorem 6.1. By Theorems 1.3 and 1.5, $\operatorname{End}\left(J\left(C_{f_{1}}\right)\right)=$ $\mathbb{Z}$ and $\left.\operatorname{End}\left(J\left(C_{f_{2}}\right)\right)\right)=\mathbb{Z}$. This implies that both jacobians $J\left(C_{f_{1}}\right)$ and $J\left(C_{f_{2}}\right)$ have exactly one principal polarization and therefore a $\bar{K}$-isomorphism of abelian varieties $J\left(C_{f_{1}}\right) \cong J\left(C_{f_{2}}\right)$ respects the principal polarizations. Now the Torelli theorem implies that the hyperelliptic curves $C_{f_{1}}$ and $C_{f_{2}}$ are isomorphic over $\bar{K}$. It follows from Theorem 6.1 that both $t_{1}$ and $t_{2}$ belong to $B(h)$.

\section{Compact groups And Simple Lie Algebras}

Lemma 7.1. Let $G$ be a compact group, $g \geq 3$ an integer and $\pi: G \rightarrow \mathbf{A}_{2 g} a$ continuous surjective group homomorphism. Let $M$ be a finite group and $\pi^{\prime}: G \rightarrow$ $M$ be a continuous group homomorphism. Let us put $H:=\operatorname{ker}\left(\pi^{\prime}\right)$. Suppose that one of the following conditions holds:

(i) $M$ is solvable.

(ii) The order of $M$ is strictly less than the order of $\mathbf{A}_{2 g}$.

(iii) The homomorphism $\pi^{\prime}$ is surjective and there does not exist a surjective group homomorphism $M \rightarrow \mathbf{A}_{2 g}$.

Then $H$ is a normal open compact subgroup of finite index in $G$ and $\pi(H)=\mathbf{A}_{2 g}$, i.e., $\pi: H \rightarrow \mathbf{A}_{2 g}$ is a surjective continuous homomorphism. 
Proof. The first assertion about $H$ is obvious. Replacing $M$ by its subgroup $\pi^{\prime}(G)$ we may and will assume that $\pi^{\prime}$ is surjective, i.e., $M=\pi^{\prime}(G)$. In particular, $G / H \cong M$.

In order to prove the existence of the homomorphism, it suffices to do the case (iii), because in both cases (i) and (ii) a surjection $M \rightarrow \mathbf{A}_{2 g}$ does not exist, since $\mathbf{A}_{2 g}$ is simple non-abelian. So, let us assume that there are no surjective group homomorphisms from $G$ to $M$. The normality of $H$ and surjectiveness of $\pi$ imply that $\pi(H)$ is normal in $\mathbf{A}_{2 g}$, i.e., either $\pi(H)=\mathbf{A}_{2 g}$ or $\pi(H)=\{1\}$. If $\pi(H)=\mathbf{A}_{2 g}$ then we are done. If $\pi(H)=\{1\}$ then $\pi$ factors through $G / H=M$ and we get a surjective homomorphism $M \rightarrow \mathbf{A}_{2 g}$.

7.2. Let $V$ be a non-zero even-dimensional $\mathbb{Q}_{\ell}$-vector space,

$$
e: V \times V \rightarrow \mathbb{Q}_{\ell}
$$

an alternating nondegenerate $\mathbb{Q}_{\ell}$-bilinear form,

$\operatorname{Sp}(V, e)=\operatorname{Aut}(V, e)=\left\{s \in \operatorname{Aut}_{\mathbb{Q}_{\ell}}(V) \mid e(s x, s y)=e(x, y) \forall x, y \in V\right\} \subset \operatorname{Aut}_{\mathbb{Q}_{\ell}}(V)$ the corresponding symplectic group, viewed as a closed $\ell$-adic Lie subgroup in $\operatorname{Aut}_{\mathbb{Q}_{\ell}}(V)$ and

$$
\begin{gathered}
\mathfrak{s p}(V, e):=\operatorname{Lie}(\operatorname{Sp}(V, e))= \\
\left\{u \in \operatorname{End}_{\mathbb{Q}_{\ell}}(V) \mid e(u x, y)+e(x, u y)=0 \forall x, y \in V\right\} \subset \operatorname{End}_{\mathbb{Q}_{\ell}}(V)
\end{gathered}
$$

its Lie algebra, viewed as a $\mathbb{Q}_{\ell}$-Lie subalgebra in $\operatorname{End}_{\mathbb{Q}_{\ell}}(V)$. It is well-known that $\operatorname{Sp}(V, e) \subset \operatorname{SL}(V), \mathfrak{s p}(V, e) \subset \operatorname{Lie}(\operatorname{SL}(V))=\mathfrak{s l}(V):=\left\{u \in \operatorname{End}_{\mathbb{Q}_{\ell}}(V) \mid \operatorname{tr}_{V}(u)=0\right\}$ where $\operatorname{tr}_{V}: \operatorname{End}_{\mathbb{Q}_{\ell}}(V) \rightarrow \mathbb{Q}_{\ell}$ is the trace map. Let us consider the group of symplectic similitudes

$$
\begin{gathered}
\operatorname{Gp}(V, e)=\left\{s \in \operatorname{Aut}_{\mathbb{Q}_{\ell}}(V) \mid \exists c \in \mathbb{Q}_{\ell}^{*} \text { such that } e(s x, s y)=c \cdot e(x, y)\right. \\
\forall x, y \in V\} \subset \operatorname{Aut}_{\mathbb{Q}_{\ell}}(V) ;
\end{gathered}
$$

it is also an $\ell$-adic Lie (sub)group, whose Lie algebra coincides with $\mathbb{Q}_{\ell} \operatorname{Id} \oplus \mathfrak{s p}(V, e)$ where Id $: V \rightarrow V$ is the identity map. The map $s \mapsto c$ defines the $\ell$-adic Lie group homomorphism

$$
\chi_{e}: \operatorname{Gp}(V, e) \rightarrow \mathbb{Q}_{\ell}^{*},
$$

whose kernel coincides with $\operatorname{Sp}(V, e)$.

Let $G$ be a compact subgroup in $\operatorname{Aut}_{\mathbb{Q}_{\ell}}(V)$. The compactness implies that $G$ is closed. By the $\ell$-adic version of Cartan's theorem [31, Part II, Ch. V, Sect. 9, p. 155], $G$ is an $\ell$-adic Lie (sub)group. We write $\operatorname{Lie}(G)$ for the Lie algebra of $G$. Then $\operatorname{Lie}(G)$ is a $\mathbb{Q}_{\ell}$-Lie subalgebra of $\operatorname{End}_{\mathbb{Q}_{\ell}}(V)$. If $G \subset \operatorname{Sp}(V, e)$ or $G \subset \operatorname{Gp}(V, e)$ then $\operatorname{Lie}(G) \subset \mathfrak{s p}(V, e)$ or $\operatorname{Lie}(G) \subset \mathbb{Q}_{\ell} \operatorname{Id} \oplus \mathfrak{s p}(V, e)$ respectively.

Lemma 7.3. Let $G$ be a compact subgroup in $\operatorname{Aut}_{\mathbb{Q}_{\ell}}(V)$. Suppose that for every open subgroup $G^{\prime} \subset G$ of finite index the $G^{\prime}$-module $V$ is absolutely simple. Then there exists a semisimple $\mathbb{Q}_{\ell}$-Lie algebra $\mathfrak{g}^{s s} \subset \operatorname{End}_{\mathbb{Q}_{\ell}}(V)$ that enjoys the following properties:

(i) $\operatorname{Lie}(G)=\mathfrak{g}^{s s}$ or $\mathbb{Q}_{\ell} \operatorname{Id} \oplus \mathfrak{g}^{s s}$.

(ii) The $\mathfrak{g}^{s s}$-module $V$ is absolutely simple.

(iii) If $G \subset \operatorname{Gp}(V, e)$ then

$$
\mathfrak{g}^{s s}=\operatorname{Lie}(G) \bigcap \mathfrak{s p}(V, e) \subset \mathfrak{s p}(V, e) .
$$

In addition, if $G^{0}=\operatorname{ker}\left(\chi_{e}: G \rightarrow \mathbb{Q}_{\ell}^{*}\right)$ then $\operatorname{Lie}\left(G^{0}\right)=\mathfrak{g}^{s s}$. 
Proof. Clearly, $\operatorname{Lie}(G)=\operatorname{Lie}\left(G^{\prime}\right)$ for all $G^{\prime}$. Since the $G$-module $V$ is (semi)simple, it follows from [27, Proposition 1] that $\operatorname{Lie}(G)$ is reductive, i.e., $\operatorname{Lie}(G)=\mathfrak{g}^{s s} \oplus \mathfrak{c}$ where $\mathfrak{c}$ is the center of $\operatorname{Lie}(G)$ and $\mathfrak{g}^{s s}$ is a semisimple $\mathbb{Q}_{\ell}$-Lie algebra. I claim that $\operatorname{End}_{\operatorname{Lie}(G)}(V)=\mathbb{Q}_{\ell}$ Id. Indeed, let $G^{\prime}$ be an open subgroup of finite index that is sufficiently small in order to lie in the image of the exponential map. Then $\operatorname{End}_{G^{\prime}}(V)=\operatorname{End}_{\text {Lie }(G)}(V)$. In light of the absolute simplicity of the $G^{\prime}$-module $V$, we have $\operatorname{End}_{G^{\prime}}(V)=\mathbb{Q}_{\ell}$ Id and therefore

$$
\operatorname{End}_{\text {Lie }(G)}(V)=\operatorname{End}_{G^{\prime}}(V)=\mathbb{Q}_{\ell} \mathrm{Id} .
$$

Since the center $\mathfrak{c}$ lies in $\operatorname{End}_{\operatorname{Lie}(G)}(V)$, either $\mathfrak{c}=\{0\}$ and $\operatorname{Lie}(G)=\mathfrak{g}^{s s}$ or $\mathfrak{c}=\mathbb{Q}_{\ell} \operatorname{Id}$ and $\operatorname{Lie}(G)=\mathbb{Q}_{\ell} \operatorname{Id} \oplus \mathfrak{g}^{s s}$. In both cases

$$
\mathbb{Q}_{\ell} \operatorname{Id}=\operatorname{End}_{\operatorname{Lie}(G)}(V)=\operatorname{End}_{\mathfrak{g}^{s s}}(V) .
$$

Since $\mathfrak{g}^{s s}$ is semisimple, the $\mathfrak{g}^{s s}$-module $V$ is absolutely simple. This proves (i) and (ii). In order to prove (iii), notice that in both cases the semisimple $\mathfrak{g}^{s s}=$ $[\operatorname{Lie}(G), \operatorname{Lie}(G)] . \quad$ Clearly, $\operatorname{Lie}(G) \subset \mathbb{Q}_{\ell} \operatorname{Id} \oplus \mathfrak{s p}(V, e)$. Taking into account that $\mathfrak{s p}(V, e)$ is a simple Lie algebra, we have

$$
\mathfrak{g}^{s s}=[\operatorname{Lie}(G), \operatorname{Lie}(G)] \subset\left[\mathbb{Q}_{\ell} \operatorname{Id} \oplus \mathfrak{s p}(V, e), \mathbb{Q}_{\ell} \operatorname{Id} \oplus \mathfrak{s p}(V, e)\right]=\mathfrak{s p}(V, e) .
$$

It follows easily that

$$
\mathfrak{g}^{s s}=\operatorname{Lie}(G) \bigcap \mathfrak{s p}(V, e) .
$$

In order to compute Lie $\left(G^{0}\right)$, notice that according to [31, Part II, Ch. V, Sect. 2, p. 131], the Lie algebra of the kernel of $\chi_{e}$ coincides with the kernel of the corresponding tangent map $\operatorname{Lie}(G) \rightarrow \operatorname{Lie}\left(\mathbb{Q}_{\ell}^{*}\right)=\mathbb{Q}_{\ell}$. It follows that either $\operatorname{Lie}\left(G^{0}\right)=\operatorname{Lie}(G)$ or $\operatorname{Lie}\left(G^{0}\right)$ is a Lie subalgebra of codimension 1 in $\operatorname{Lie}(G)$. On the other hand, since $G^{0}=\operatorname{Sp}(V, e) \bigcap G$, we have

$$
\operatorname{Lie}\left(G^{0}\right) \subset \operatorname{Lie}(G) \bigcap \mathfrak{s p}(V, e)=\mathfrak{g}^{s s} .
$$

So, if $\operatorname{Lie}(G)=\mathbb{Q}_{\ell} \operatorname{Id} \oplus \mathfrak{g}^{s s}$ then the (co)dimension arguments imply that $\operatorname{Lie}\left(G^{0}\right)=$ $\mathfrak{g}^{s s}$. If $\operatorname{Lie}(G)=\mathfrak{g}^{s s}$ then the tangent map is the zero map, because $\operatorname{Lie}(G)$ is semisimple and $\mathbb{Q}_{\ell}$ is commutative. This implies that the kernel of the tangent map coincides with the whole $\operatorname{Lie}(G)$, i.e.,

$$
\operatorname{Lie}\left(G^{0}\right)=\operatorname{Lie}(G)=\mathfrak{g}^{s s} .
$$

Theorem 7.4. Let $g \geq 5$ be an integer, $V$ a $2 g$-dimensional vector space over $\mathbb{Q}_{2}$. Let $G \subset \mathrm{SL}(V) \subset \operatorname{Aut}_{\mathbb{Q}_{2}}(V)$ be a compact 2-adic Lie group that enjoys the following properties:

(i) There exists a continuous surjective homomorphism $\pi: G \rightarrow \mathbf{A}_{2 g}$;

(ii) Let $\mathfrak{g} \subset \operatorname{End}_{\mathbb{Q}_{2}}(V)$ be the $\mathbb{Q}_{2}$-Lie algebra of $G$. Then the $\mathfrak{g}$-module $V$ is absolutely simple, i.e., the natural representation of $\mathfrak{g}$ in $V$ is irreducible and $\operatorname{End}_{\mathfrak{g}}(V)=\mathbb{Q}_{2}$.

Then:

(i) The $\mathbb{Q}_{2}$-Lie algebra $\mathfrak{g}$ is absolutely simple.

(ii) Let $L / \mathbb{Q}_{2}$ be a finite Galois field extension such that the L-Lie algebra $\mathfrak{g}_{L}:=$ $\mathfrak{g} \otimes \mathbb{Q}_{2} L$ is split. (Such $L$ always exists.) If $W$ is a faithful simple $\mathfrak{g}_{L}$-module of finite L-dimension then $\operatorname{dim}_{L}(W) \geq 2 g-2$. 
Proof. Our plan is to apply Corollary 2.5 to a certain compact 2-adic Lie group that will be obtained from $G$ in several steps. At every step we replace the group either by the kernel of a homomorphism to a "small" finite group or by the image of a homomorphism, whose kernel is a pro-2-group.

Since $G \subset \mathrm{SL}(V)$, we have $\mathfrak{g} \subset \mathfrak{s l}(V)$. Clearly, $\mathfrak{g}$ is reductive, its center is either $\{0\}$ or the scalars. Since $\mathfrak{g} \subset \mathfrak{s l}(V)$, the center of $\mathfrak{g}$ is $\{0\}$. This implies that $\mathfrak{g}$ is semisimple. Let $\mathfrak{G} \subset \mathrm{GL}(V)$ be the connected semisimple linear algebraic (sub)group over $\mathbb{Q}_{2}$, whose Lie algebra coincides with $\mathfrak{g}$.

Let us prove that the semisimple $\mathbb{Q}_{2}$-Lie algebra $\mathfrak{g}$ is, in fact, absolutely simple. Indeed, there exists a finite Galois field extension $L / \mathbb{Q}_{2}$ such that the semisimple $L$-Lie algebra $\mathfrak{g}_{L}=\mathfrak{g} \otimes_{\mathbb{Q}_{2}} L$ is split; in particular, $\mathfrak{g}_{L}$ splits into a (finite) direct sum

$$
\mathfrak{g}_{L}=\oplus_{i \in I} g_{i}
$$

of absolutely simple split $L$-Lie algebras $\mathfrak{g}_{i}$. Here $I$ is the set of minimal ideals $\mathfrak{g}_{i}$ in $\mathfrak{g}$. It is well-known that the $L$-vector space

$$
V_{L}=V \otimes_{\mathbb{Q}_{2}} L
$$

becomes an absolutely simple faithful $\mathfrak{g}_{L}$-module and splits into a tensor product

$$
V_{L}=\otimes_{i \in I} W_{i}
$$

of absolutely simple faithful $\mathfrak{g}_{i}$-modules $W_{i}$. Since each $\mathfrak{g}_{i}$ is simple and $W_{i}$ is faithful,

$$
\operatorname{dim}_{L}\left(W_{i}\right) \geq 2 \forall i \in I
$$

and therefore

$$
n:=\#(I) \leq \log _{2}\left(\operatorname{dim}_{L}\left(V_{L}\right)\right)=\log _{2}\left(\operatorname{dim}_{\mathbb{Q}_{2}}(V)\right)=\log _{2}(2 g)<g .
$$

Let us consider the adjoint representation

$$
\operatorname{Ad}: G \rightarrow \operatorname{Aut}(\mathfrak{g}) \subset \operatorname{Aut}\left(\mathfrak{g}_{L}\right) .
$$

Since the $\mathfrak{g}$-module $V$ is absolutely simple, $\operatorname{ker}(\mathrm{Ad})$ consists of scalars. Since $G \subset$ $\mathrm{SL}(V)$, the group $\operatorname{ker}(\mathrm{Ad})$ is finite commutative. Clearly, $G$ permutes elements of $I$ and therefore gives rise to the continuous homomorphism (composition)

$$
\pi_{1}: G \stackrel{\operatorname{Ad}}{\rightarrow} \operatorname{Aut}(\mathfrak{g}) \subset \operatorname{Aut}\left(\mathfrak{g}_{L}\right) \rightarrow \operatorname{Perm}(I) \cong \mathbf{S}_{n} .
$$

Let $G_{1}$ be the kernel of $\pi_{1}$ : it is an open normal subgroup of finite index in $G$ and therefore the Lie algebra of $G_{1}$ coincides with $\mathfrak{g}$. Since $n<g$,

$$
\#\left(\mathbf{S}_{n}\right)=n !<\frac{1}{2}(2 g) !=\#\left(\mathbf{A}_{2 g}\right) .
$$

It follows from Lemma 7.1 applied to $\pi^{\prime}=\pi_{1}$ and $M=\mathbf{S}_{n}$, that $\pi\left(G_{1}\right)=\mathbf{A}_{2 g}$. So, we may replace $G$ by $G_{1}$ and assume that $G$ leaves stable every $g_{i}$. This means that the image of

$$
G \stackrel{\operatorname{Ad}}{\rightarrow} \operatorname{Aut}\left(\mathfrak{g}_{L}\right)=\operatorname{Aut}\left(\oplus_{i \in I} \mathfrak{g}_{i}\right)
$$

lies in $\prod_{i \in I} \operatorname{Aut}\left(\mathfrak{g}_{i}\right)$.

We write $\mathfrak{G}_{L} \subset \mathrm{GL}\left(V_{L}\right)$ for the connected semisimple linear algebraic (sub)group over $L$ obtained from $\mathfrak{G}$ by extension of scalars. Clearly, the $L$-Lie algebra of $\mathfrak{G}_{L}$ coincides with $\mathfrak{g}_{L}$. We write $\mathfrak{G}_{i}$ for the simply-connected absolutely simple split $L$ algebraic group, whose Lie algebra coincides with $\mathfrak{g}_{i}[34]$. We write $\mathfrak{G}^{\mathrm{Ad}} \subset \mathrm{GL}\left(\mathfrak{g}_{L}\right)$ 
for the adjoint group of $\mathfrak{G}$ and $\operatorname{Ad}_{\mathfrak{G}}$ for the corresponding central isogeny $\mathfrak{G} \rightarrow \mathfrak{G}^{\mathrm{Ad}}$. If $\mathfrak{G}_{i}^{\mathrm{Ad}} \subset \mathrm{GL}\left(\mathfrak{g}_{i}\right)$ is the adjoint group of $\mathfrak{G}_{i}$ then

$$
\mathfrak{G}^{\mathrm{Ad}}=\prod_{i \in I} \mathfrak{G}_{i}^{\mathrm{Ad}} .
$$

Since $g_{i}$ is simple split, the group $\mathfrak{G}_{i}^{\mathrm{Ad}}(L)$ is a closed (in the 2-adic topology) normal subgroup in $\operatorname{Aut}\left(\mathfrak{g}_{i}\right)$ of index 1, 2 or 6 [15, Ch. 9, Sect. 4, Th. 4 and Remark on p. 281]. Let us consider the composition

$$
G \stackrel{\operatorname{Ad}}{\rightarrow} \prod_{i \in I} \operatorname{Aut}\left(\mathfrak{g}_{i}\right) \rightarrow \prod_{i \in I} \operatorname{Aut}\left(\mathfrak{g}_{i}\right) / \mathfrak{G}_{i}^{\operatorname{Ad}}(L) .
$$

Its image is a finite solvable group. Let $G_{2}$ be its kernel: it is an open normal subgroup of finite index in $G$ and the Lie algebra of $G_{1}$ coincides with $\mathfrak{g}$. It follows from Lemma 7.1 applied to $\pi^{\prime}=\pi_{2}$ that $\pi\left(G_{2}\right)=\mathbf{A}_{2 g}$. So, we may replace $G$ by $G_{2}$ and assume that the image of $G \stackrel{\text { Ad }}{\rightarrow} \prod_{i \in I} \operatorname{Aut}\left(\mathfrak{g}_{i}\right)$ lies in $\prod_{i \in I} \mathfrak{G}_{i}^{\text {Ad }}(L)$. So, we get the continuous group homomorphism

$$
\gamma: G \stackrel{\operatorname{Ad}}{\rightarrow} \prod_{i \in I} \mathfrak{G}_{i}^{\operatorname{Ad}}(L)=\mathfrak{G}^{\operatorname{Ad}}(L) .
$$

Recall that $\operatorname{ker}(\mathrm{Ad})$ is finite commutative. This implies that $\operatorname{ker}(\gamma)$ is a finite commutative group. On the other hand, let us consider the canonical central isogeny of semisimple $L$-algebraic groups

$$
\alpha:=\operatorname{Ad}_{\mathfrak{G}}: \mathfrak{G}=\prod_{i \in I} \mathfrak{G}_{i} \rightarrow \prod_{i \in I} \mathfrak{G}_{i}^{\mathrm{Ad}}=\mathfrak{G}^{\mathrm{Ad}} .
$$

Applying [42, Corollary 3.4(2) on p. 409], we conclude that there exists a compact subgroup $G_{3} \subset \mathfrak{G}(L)=\prod_{i \in I} \mathfrak{G}_{i}(L)$ and a surjective continuous homomorphism $\pi_{3}: G_{3} \rightarrow \mathbf{A}_{2 g}$, whose kernel $H$ is an open normal subgroup of finite index in $G_{3}$. Applying [42, Prop. 3.3 on pp. $408-409]^{2}$ to $\ell=2, F=L, G=G_{3}, S_{i}=\mathfrak{G}_{i}(L)$, we conclude that there exist $j \in I$ and a compact subgroup $G_{4} \subset \mathfrak{G}_{i}(L)$ provided with surjective continuous homomorphism $G_{4} \rightarrow \mathbf{A}_{2 g}$.

Let $W$ be a finite-dimensional $L$-vector space that carries the structure of absolutely simple faithful $\mathfrak{g}$-module. I claim that

$$
\operatorname{dim}_{L}(W) \geq 2 g-2 \text {. }
$$

Indeed, there exists a $L$-rational representation

$$
\rho_{W}: \mathfrak{G}_{i} \rightarrow \mathrm{GL}(W),
$$

whose kernel is a finite central subgroup. The composition

$$
\pi_{4}: G_{4} \subset \mathfrak{G}_{i}(L) \stackrel{\rho_{W}}{\rightarrow} \operatorname{Aut}_{L}(W)
$$

is a continuous group homomorphism, whose kernel is a finite central subgroup of $G_{4}$. Clearly, the central subgroup $\operatorname{ker}\left(\pi_{4}\right)$ is killed by the surjective homomorphism $G_{4} \rightarrow \mathbf{A}_{2 g}$. So, if we put $G_{5}=\pi_{4}\left(G_{4}\right) \subset \operatorname{Aut}_{L}(W)$ then $G_{5}$ is a compact subgroup that admits a surjective continuous homomorphism $G_{5} \rightarrow \mathbf{A}_{2 g}$. It follows from Corollary 2.5 that

$$
\operatorname{dim}_{L}(W) \geq 2 g-2 \text {. }
$$

\footnotetext{
${ }^{2}$ The $R_{j}$ in $\left[42\right.$, p. 409 , line 3] should be $S_{j}$.
} 
In particular, $\operatorname{dim}_{L}\left(V_{j}\right) \geq 2 g-2$. On the other hand,

$$
\begin{gathered}
2 g=\operatorname{dim}_{\mathbb{Q}_{2}}(V)=\operatorname{dim}_{L}\left(V_{L}\right)=\prod_{i \in I} \operatorname{dim}_{L}\left(V_{i}\right)= \\
\operatorname{dim}_{L}\left(V_{j}\right) \prod_{i \in I, i \neq j} \operatorname{dim}_{L}\left(V_{i}\right) \geq(2 g-2) 2^{\#(I)-1} .
\end{gathered}
$$

It follows that $\#(I)=1$, i.e., $I=\{j\}$ and $\mathfrak{g}_{L}=\mathfrak{g}_{j}$. This means that $\mathfrak{g}_{L}$ is an absolutely simple $L$-Lie algebra and therefore $\mathfrak{g}$ is an absolutely simple $\mathbb{Q}_{2}$-Lie algebra.

Corollary 7.5. Let $g \geq 5$ be an integer, $V$ a $2 g$-dimensional vector space over $\mathbb{Q}_{2}$ and

$$
e: V \times V \rightarrow \mathbb{Q}_{2}
$$

an alternating nondegenerate $\mathbb{Q}_{2}$-bilinear form. Let $G \subset \operatorname{Gp}(V, e) \subset \operatorname{Aut}_{\mathbb{Q}_{2}}(V)$ be a compact 2-adic Lie group that enjoys the following properties:

(i) For every open subgroup $G^{\prime} \subset G$ of finite index the $G^{\prime}$-module $V$ is absolutely simple.

(ii) There exists a continuous surjective homomorphism $\pi: G \rightarrow \mathbf{A}_{2 g}$.

Let $G^{0}$ be the kernel of $\chi_{e}: G \rightarrow \mathbb{Q}_{\ell}^{*}$ and

$$
\mathfrak{g}:=\operatorname{Lie}\left(G^{0}\right) \subset \operatorname{End}_{\mathbb{Q}_{2}}(V) .
$$

Then:

(i) There exists a continuous surjective homomorphism $\pi: G^{0} \rightarrow \mathbf{A}_{2 g}$.

(ii) The $\mathbb{Q}_{2}$-Lie algebra $\mathfrak{g}:=\operatorname{Lie}\left(G^{0}\right)$ is absolutely simple and the $\mathfrak{g}$-module $V$ is absolutely simple. In addition, $\mathfrak{g}=\operatorname{Lie}(G) \cap \mathfrak{s p}(V, e)$ and the $\mathfrak{g}$-module $V$ is symplectic.

(iii) Either $\operatorname{Lie}(G)=\mathfrak{g}$ or $\operatorname{Lie}(G)=\mathbb{Q}_{2} \operatorname{Id} \oplus \mathfrak{g}$.

(iv) Let $L / \mathbb{Q}_{2}$ be a finite Galois field extension such that the simple L-Lie algebra $\mathfrak{g}_{L}=\mathfrak{g} \otimes_{\mathbb{Q}_{2}} L$ is split. If $W$ is a faithful simple $\mathfrak{g}_{L}$-module of finite $L$ dimension then $\operatorname{dim}_{L}(W) \geq 2 g-2$.

Proof. In order to prove (i), notice that $\pi\left(G_{0}\right)$ is a normal subgroup in $\mathbf{A}_{2 g}$, i.e., $\pi\left(G_{0}\right)$ is either $\mathbf{A}_{2 g}$ or $\{1\}$. In the former case we are done, so let us assume that $\pi\left(G_{0}\right)=\{1\}$. This means that $\pi$ kills $G_{0}$ and therefore induces a surjective homomorphism $G / G_{0} \rightarrow \mathbf{A}_{2 g}$. On the other hand, $G / G_{0}$ is isomorphic to the subgroup $\chi_{e}(G)$ of $\mathbb{Q}_{\ell}^{*}$ and therefore is commutative. It follows that $\mathbf{A}_{2 g}$ is also commutative, which is not the case. This contradiction proves (i).

Lemma 7.3 implies all the assertions in (ii) and (iii) except the absolute simplicity of $\mathfrak{g}^{s s}=\mathfrak{g}$; however, it implies that $\mathfrak{g}$ is semisimple and that the $\mathfrak{g}$-module $V$ is absolutely simple. Now the the absolute simplicity of $\mathfrak{g}$ follows from Theorem 7.4 applied to $G^{0}$. The assertion (iv) also follows from Theorem 7.4 applied to $G^{0}$.

We refer to [3, Ch. VIII, Sect. 7.3] for the notion and basic properties of minuscule weights. (See also [28] and [37].)

Corollary 7.6. We keep all notation and assumption of Corollary 7.5. Let $L / \mathbb{Q}_{2}$ be a finite Galois field extension such that $\mathfrak{g}_{L}$ is split. Assume also that $\mathfrak{g}_{L}$ is a classical simple Lie algebra, the $\mathfrak{g}_{L}$-module $V_{L}=V \otimes_{\mathbb{Q}_{2}} L$ is fundamental and its highest weight is a minuscule weight. 
Then $\mathfrak{g}=\mathfrak{s p}(V, e)$. In particular, $\mathfrak{g}$ is an absolutely simple $\mathbb{Q}_{2}$-Lie algebra of of type $\mathrm{C}_{g}$.

Proof. In the course of the proof we will freely use Tables from [3]. It follows from Corollary 7.5 and Theorem 7.4 that if $W$ is a faithful simple $\mathfrak{g}_{L}$-module of finite $L$-dimension then $\operatorname{dim}_{L}(W) \geq 2 g-2$.

Extending the form $e$ by $L$-linearity to $V_{L}$, we obtain the alternating nondegenerate $L$-bilinear form

$$
e_{L}: V_{L} \times V_{L} \rightarrow L
$$

Clearly,

$$
\mathfrak{g}_{L} \subset \mathfrak{s p}\left(V_{L}, e_{L}\right) ;
$$

in particular, $V_{L}$ is the symplectic $\mathfrak{g}_{L}$-module.

Let $r$ be the rank of the absolutely simple classical $L$-Lie algebra $\mathfrak{g}_{L}$.

If $\mathfrak{g}_{L}$ is of type $\mathrm{A}_{r}$ then there exists a $(r+1)$-dimensional $L$-vector space $W$ such that $\mathfrak{g}_{L} \cong \mathfrak{s l}(W)$ and the fundamental $\mathfrak{g}_{L}$-module $V_{L}$ is isomorphic to $\wedge_{L}^{i}(W)$ for suitable $i$ with $1 \leq i \leq r$. However,

$$
r+1=\operatorname{dim}_{L}(W) \geq 2 g-2 \geq 8 ;
$$

in particular, $r \geq 8$. Since $V_{L}$ is symplectic,

$$
2 \leq i \leq r-1 .
$$

We have

$$
\begin{gathered}
\left.2 g=\operatorname{dim}_{\mathbb{Q}_{2}}(V)=\operatorname{dim}_{L}\left(V_{L}\right)\right)=\operatorname{dim}_{L}\left(\wedge_{L}^{i}(W)\right) \geq \operatorname{dim}_{L}\left(\wedge_{L}^{2}(W)=\frac{(r+1) r}{2} \geq\right. \\
4(r+1)>(r+1)+4>(2 g-2)+3>2 g .
\end{gathered}
$$

The obtained contradiction proves that $\mathfrak{g}$ is not of type $\mathrm{A}_{r}$.

If $\mathfrak{g}_{L}$ is of type $\mathrm{B}_{r}$ then there exists a $(2 r+1)$-dimensional $L$-vector space $W$ such that $W$ is an (orthogonal) absolutely simple faithful $\mathfrak{g}_{L}$-module and every absolutely simple $\mathfrak{g}_{L}$-module with minuscule highest weight has dimension $2^{r}$. Hence

$$
\operatorname{dim}_{L}\left(V_{L}\right)=2^{r} .
$$

We have

$$
2 g=\operatorname{dim}_{L}\left(V_{L}\right)=2^{r}, 2 r+1=\operatorname{dim}_{L}(W) \geq 2 g-2 .
$$

This implies that $g=2^{r-1}$ and therefore $r>3$, since $g \geq 5$. We also have $2 r+1=\operatorname{dim}_{L}(W) \geq 2 g-2=2^{r}-2$, which implies that $2 r+1 \geq 2^{r}-2$. It follows that $r \leq 3$ and therefore $g=2^{r-1} \leq 4$, which could not be the case. The obtained contradiction proves that $\mathfrak{g}$ is not of type $\mathrm{B}_{r}$.

If $\mathfrak{g}_{L}$ is of type $\mathrm{C}_{r}$ then the only absolutely simple (symplectic) $\mathfrak{g}_{L}$-module, with minuscule highest weight has dimension $2 r$. This implies that $\operatorname{dim}_{L}\left(V_{L}\right)=2 r$ and therefore $\operatorname{dim}_{L}\left(\mathfrak{g}_{L}\right)=\operatorname{dim}_{L}\left(\mathfrak{s p}\left(V_{L}, e_{L}\right)\right)$. Since $\mathfrak{g}_{L} \subset \mathfrak{s p}\left(V_{L}, e_{L}\right)$, we conclude that

$$
\mathfrak{g}_{L}=\mathfrak{s p}\left(V_{L}, e_{L}\right) \subset \operatorname{End}_{L}\left(V_{L}\right) .
$$

Now the dimension arguments imply that $\mathfrak{g}=\mathfrak{s p}(V, e)$.

If $\mathfrak{g}_{L}$ is of type $\mathrm{D}_{r}$ (with $r \geq 3$ ) then there exists a $2 r$-dimensional $L$-vector space $W$ such that $W$ is an (orthogonal) absolutely simple faithful $\mathfrak{g}_{L}$-module; on the other hand, every absolutely simple symplectic $\mathfrak{g}_{L}$-module, with minuscule highest weight must have dimension $2^{r-1}$. This implies that

$$
\operatorname{dim}_{L}\left(V_{L}\right)=2^{r-1} .
$$


We have $2 g=\operatorname{dim}_{L}\left(V_{L}\right)=2^{r-1}$ and therefore $g=2^{r-2}$. Since $g \geq 5$ we have $r \geq 5$. We have

$$
2 r=\operatorname{dim}_{L}(W) \geq 2 g-2=2^{r-1}-2
$$

and therefore $2 r \geq 2^{r-1}-2$, which could not be the case, since $r \geq 5$. The obtained contradiction proves that $\mathfrak{g}$ is not of type $\mathrm{D}_{r}$.

\section{Abelian VARIETIES Without NONTRIVIAL ENDOMORPHISMS}

We use the notation of Sections 3 and 4 . Let $F$ be a field and $\ell$ a prime different from $\operatorname{char}(F)$. As usual, we write $\mathbb{Z}_{\ell}(1)$ for the projective limit of the cyclic multiplicative groups

$$
\mu_{\ell^{i}}:=\left\{z \in \bar{F}^{*} \mid z^{\ell^{i}}=1\right\} .
$$

The group $\mathbb{Z}_{\ell}(1)$ carries the natural structure of free $\mathbb{Z}_{\ell}$-module of rank 1 . It also carries the natural structure of the Galois module provided by the $\ell$-adic cyclotomic character

$$
\chi_{\ell}: \operatorname{Gal}(K) \rightarrow \mathbb{Z}_{\ell}^{*}=\operatorname{Aut}_{\mathbb{Z}_{\ell}}\left(\mathbb{Z}_{\ell}(1)\right) .
$$

Let us fix a (non-canonical) isomorphism of $\mathbb{Z}_{\ell}$-modules

$$
\mathbb{Z}_{\ell}(1) \cong \mathbb{Z}_{\ell}
$$

Let $X$ be an abelian variety of positive dimension over $F$ and let $\lambda$ be a polarization on $X$ that is defined over $F$. Then $\lambda$ gives rise to a Riemann form [20, Sect. $20]$

$$
e_{\lambda}: T_{\ell}(X) \times T_{\ell}(X) \rightarrow \mathbb{Z}_{\ell}(1) \cong \mathbb{Z}_{\ell}
$$

$e_{\lambda}$ is a nondegenerate $\operatorname{Gal}(K)$-equivariant alternating $\mathbb{Z}_{\ell^{-}}$bilinear form. Here the equivariance means that

$$
e_{\lambda}(\sigma(x), \sigma(y))=\chi_{\ell}(\sigma) \cdot e_{\lambda}(x, y) \forall \sigma \in \operatorname{Gal}(K) .
$$

Extending $e_{\lambda}$ by $\mathbb{Q}_{\ell}$-linearity, we obtain a nondegenerate $\operatorname{Gal}(K)$-equivariant alternating $\mathbb{Q}_{\ell}$-bilinear form

$$
e_{\lambda}: V_{\ell}(X) \times V_{\ell}(X) \rightarrow \mathbb{Q}_{\ell}
$$

such that

$$
e_{\lambda}(\sigma(x), \sigma(y))=\chi_{\ell}(\sigma) \cdot e_{\lambda}(x, y) \forall x, y \in V_{\ell}(X), \sigma \in \operatorname{Gal}(K) .
$$

This implies that

$$
G_{\ell, X}=G_{\ell, X, F}=\rho_{\ell, X}(\operatorname{Gal}(K)) \subset \operatorname{Gp}\left(V_{\ell}(X), e_{\lambda}\right) .
$$

We write $\mathfrak{g}_{\ell, X} \subset \operatorname{End}_{\mathbb{Q}_{\ell}}\left(V_{\ell}(X)\right)$ for the $\mathbb{Q}_{\ell}$-Lie algebra of the compact $\ell$-adic Lie group $G_{\ell, X, F}$. Clearly,

$$
\mathfrak{g}_{\ell, X} \subset \operatorname{Lie}\left(\operatorname{Gp}\left(V_{\ell}(X), e_{\lambda}\right)\right)=\mathbb{Q}_{\ell} \operatorname{Id} \oplus \mathfrak{s p}\left(V_{\ell}(X), e_{\lambda}\right) .
$$

Remark 8.1. Let $m$ be a positive integer and $X^{m}$ is the $m$ th power (self-product) of $X$. Then

$$
V_{\ell}\left(X^{m}\right)=\bigoplus_{j=1}^{m} V_{\ell}(X)=: V_{\ell}(X)^{m}
$$

(the equality of Galois modules) and

$$
\mathfrak{g}_{\ell, X^{m}}=\mathfrak{g}_{\ell, X} \subset \operatorname{End}_{\mathbb{Q}_{\ell}}\left(V_{\ell}(X)\right) \subset \mathrm{M}_{m}\left(\operatorname{End}_{\mathbb{Q}_{\ell}}\left(V_{\ell}(X)\right)\right)=\operatorname{End}_{\mathbb{Q}_{\ell}}\left(V_{\ell}(X)^{m}\right)
$$


(the last embedding is the diagonal one). The polarization $\lambda$ gives rise to the polarization $\lambda^{m}$ on $X^{m}$ [47, Sect. 1.12, pp. 320-321] such that the corresponding Riemann form

$$
e_{\lambda^{m}}: V_{\ell}(X)^{m} \times V_{\ell}(X)^{m} \rightarrow \mathbb{Q}_{\ell}
$$

is the direct orthogonal sum of $m$ copies of $e_{\lambda}$. Clearly, $e_{\lambda^{m}}$ is $\mathfrak{s p}\left(V_{\ell}(X), e_{\lambda}\right)$ invariant, i.e.,

$$
e_{\lambda^{m}}(u x, y)+e_{\lambda^{m}}(x, u y)=0 \quad \forall u \in \mathfrak{s p}\left(V_{\ell}(X), e_{\lambda}\right) ; x, y \in V_{\ell}(X)^{m} .
$$

It is also clear that every alternating $\mathfrak{s p}\left(V_{\ell}(X), e_{\lambda}\right)$-invariant bilinear form on $V_{\ell}(X)^{m}$ is of the form $e_{\lambda^{m}}(B x, y)$ where

$$
B \in \mathrm{M}_{m}\left(\mathbb{Q}_{\ell}\right) \subset \mathrm{M}_{m}\left(\operatorname{End}_{\mathbb{Q}_{\ell}}\left(V_{\ell}(X)\right)\right)=\operatorname{End}_{\mathbb{Q}_{\ell}}\left(V_{\ell}(X)^{m}\right)
$$

is a symmetric square matrix of size $m$ with entries from $\mathbb{Q}_{\ell}$.

Theorem 8.2. Suppose that $F$ is a field that is finitely generated over $\mathbb{Q}$. Suppose that $X$ is an abelian variety of positive dimension $g$ over $F$. Assume additionally that $X$ enjoys the following properties:

- $\operatorname{End}(X)=\mathbb{Z}$.

- $\tilde{G}_{2, X, F}$ is isomorphic either to $\mathbf{S}_{2 g}$ or to $\mathbf{A}_{2 g}$.

- $g \geq 5$.

Then $\mathfrak{g}_{\ell, X}=\mathbb{Q}_{\ell} \operatorname{Id} \oplus \mathfrak{s p}\left(V_{\ell}(X), e_{\lambda}\right)$ for all $\ell$. In particular, $G_{\ell, X, F}$ is an open subgroup in $\operatorname{Gp}\left(V_{\ell}(X), e_{\lambda}\right)$.

Proof. The openness property follows from the coincidence of the corresponding $\mathbb{Q}_{\ell}$-Lie algebras. So, it is suffices to check that $\mathfrak{g}_{\ell, X}=\mathbb{Q}_{\ell} \operatorname{Id} \oplus \mathfrak{s p}\left(V_{\ell}(X), e_{\lambda}\right)$.

Replacing if necessary, $F$ by its suitable quadratic extension, we may and will assume that $\tilde{G}_{2, X, F}=\mathbf{A}_{2 g}$.

By a theorem of Bogomolov [1, 2],

$$
\mathfrak{g}_{\ell, X} \supset \mathbb{Q}_{\ell} \mathrm{Id} .
$$

By a theorem of Faltings $[9,10]$ for every finite algebraic extension $F_{1}$ of $F$ the $\operatorname{Gal}\left(F_{1}\right)$-module $V_{\ell}$ is semisimple and

$$
\operatorname{End}_{\mathrm{Gal}\left(F_{1}\right)}\left(V_{\ell}(X)\right)=\operatorname{End}_{F_{1}}(X) \otimes \mathbb{Q}_{\ell}=\mathbb{Z} \otimes \mathbb{Q}_{\ell}=\mathbb{Q}_{\ell} .
$$

In other words, the $\operatorname{Gal}\left(F_{1}\right)$-module $V_{\ell}$ is absolutely simple. Notice that $\rho_{\ell, X}\left(\operatorname{Gal}\left(F_{1}\right)\right)$ is an open subgroup of finite index in $G_{\ell, X, F}$. Conversely, every open subgroup of finite index in $G_{\ell, X, F}$ coincides with $\rho_{\ell, X}\left(\operatorname{Gal}\left(F_{1}\right)\right)$ for some finite algebraic extension $F_{1}$ of $F$. It follows that the $\mathbb{Q}_{\ell}$-Lie algebra $\mathfrak{g}_{\ell, X}$ is reductive and the $\mathfrak{g}_{\ell, X}$-module $V_{\ell}(X)$ is absolutely simple, i.e.,

$$
\operatorname{End}_{\mathfrak{g}_{\ell, X}}\left(V_{\ell}(X)\right)=\mathbb{Q}_{\ell} .
$$

It follows from Lemma 7.3 that $\mathfrak{g}_{\ell, X}=\mathbb{Q}_{\ell} \operatorname{Id} \oplus \mathfrak{g}_{\ell}^{s s}$ where $\mathfrak{g}_{\ell}^{s s}$ is a semisimple $\mathbb{Q}_{\ell}$-Lie algebra such that

$$
\mathfrak{g}_{\ell}^{s s} \subset \mathfrak{s p}\left(V_{\ell}(X), e_{\lambda}\right)
$$

and the $\mathfrak{g}_{\ell}^{s s}$-module $V_{\ell}(X)$ is absolutely simple. Pick a finite algebraic field extension $L / \mathbb{Q}_{\ell}$ such that the semisimple $L$-Lie algebra $\mathfrak{g}=\mathfrak{g}_{\ell}^{s s} \otimes_{\mathbb{Q}_{\ell}} L$ splits. By a theorem of Pink [23], all simple factors of $\mathfrak{g}$ are classical Lie algebras and the highest weight of the simple $\mathfrak{g}$-module $V_{\ell}(X) \otimes_{\mathbb{Q}_{\ell}} L$ is minuscule.

Now let us consider the case of $\ell=2$. Applying Corollary 7.5, we conclude that $\mathfrak{g}_{2}^{s s}$ is an absolutely simple $\mathbb{Q}_{2}$-Lie algebra and therefore $\mathfrak{g}$ is an absolutely 
simple $L$-Lie algebra. It follows from the theorem of Pink that $\mathfrak{g}$ is a classical simple Lie algebra and the highest weight of the simple $\mathfrak{g}$-module $V_{2}(X) \otimes_{\mathbb{Q}_{2}} L$ is minuscule. Applying Corollary 7.6, we conclude that $\mathfrak{g}_{2}^{s s}=\mathfrak{s p}\left(V_{2}(X), e_{\lambda}\right)$ and $\mathfrak{g}_{2, X}=\mathbb{Q}_{2} \operatorname{Id} \oplus \mathfrak{s p}\left(V_{2}(X), e_{\lambda}\right)$.

Now the case of arbitrary $\ell$ follows from Lemma 8.2 in [42].

Theorem 8.3. Suppose that $K$ is a field that is finitely generated over $\mathbb{Q}$. Suppose that $f(x) \in K[x]$ is a polynomial of degree $n \geq 10$ such that $f(x)=(x-t) h(x)$ with $t \in K$ and $h(x) \in K[x]$. Suppose that $\operatorname{Gal}(h)$ is either the full symmetric group $\mathbf{S}_{n-1}$ or the alternating group $\mathbf{A}_{n-1}$. Let $C_{f}$ be the hyperelliptic curve $y^{2}=f(x)$, let $J\left(C_{f}\right)$ be its jacobian and $\lambda$ the principal polarization on $J\left(C_{f}\right)$ attached to the theta divisor. Then for all primes $\ell$

$$
\mathfrak{g}_{\ell, J\left(C_{f}\right)}=\mathbb{Q}_{\ell} \operatorname{Id} \oplus \mathfrak{s p}\left(V_{\ell}\left(J\left(C_{f}\right)\right), e_{\lambda}\right)
$$

and the group $G_{\ell, J\left(C_{f}\right), K}$ is an open subgroup in $\operatorname{Gp}\left(V_{\ell}(X), e_{\lambda}\right)$.

Proof. As above, the openness property follows from the coincidence of the corresponding Lie algebras. So, it is suffices to check that $\mathfrak{g}_{\ell, J\left(C_{f}\right)}$ coincides with $\mathbb{Q}_{\ell} \operatorname{Id} \oplus \mathfrak{s p}\left(V_{\ell}\left(J\left(C_{f}\right)\right), e_{\lambda}\right)$.

Suppose that $n$ is even. Then as in the Proof of Theorem 1.3 (Sect. 1), the curve $C_{f}$ is $K$-biregularly isomorphic to

$$
C_{h_{2}}: y_{1}^{2}=h_{2}\left(x_{1}\right)
$$

where $h_{2}\left(x_{1}\right) \in K\left[x_{1}\right]$ is a certain degree $(n-1)$ polynomial, whose Galois group is either $\mathbf{S}_{n-1}$ or $\mathbf{A}_{n-1}$ respectively. Since $n-1 \geq 9$, the assertion follows from Theorem 2.4 of [42] applied to the polynomial $h_{2}\left(x_{1}\right)$.

Suppose now that $n$ is odd and therefore $n \geq 11$. We have $n-1=2 g$ where $g$ is an integer that is greater or equal than 5. Replacing if necessary, $K$ by its suitable quadratic extension, we may and will assume that $\operatorname{Gal}(h)=\mathbf{A}_{2 g}$. By Theorem 1.5, $\operatorname{End}\left(J\left(C_{f}\right)\right)=\mathbb{Z}$. By Lemma 5.2, $\tilde{G}_{2, J\left(C_{f}\right), \ell}=\operatorname{Gal}(h)$ and therefore

$\tilde{G}_{2, J\left(C_{f}\right), K}=\mathbf{A}_{2 g}$. As we have seen in Section 4, there is a continuous surjective homomorphism

$$
\pi_{2, J\left(C_{f}\right), K}: G_{2, J\left(C_{f}\right), K} \rightarrow \tilde{G}_{2, J\left(C_{f}\right), K}=\mathbf{A}_{2 g} .
$$

Now the result follows from Theorem 8.2 applied to $F=K$ and $X=J\left(C_{f}\right)$.

\section{TAte Classes}

The aim of this and next Sections is to use the classical invariant theory of symplectic groups [13] in order to deduce from results of the previous Section the validity of certain important conjectures for our $J\left(C_{f}\right)$ and its self-products.

Theorem 9.1. Suppose that $K$ is a field that is finitely generated over $\mathbb{Q}$. Suppose that $f(x) \in K[x]$ is a polynomial of degree $n \geq 10$ such that $f(x)=(x-t) h(x)$ with $t \in K$ and $h(x) \in K[x]$. Suppose that $\operatorname{Gal}(h)$ is either the full symmetric group $\mathbf{S}_{n-1}$ or the alternating group $\mathbf{A}_{n-1}$.

Let $C_{f}$ be the hyperelliptic curve $y^{2}=f(x)$ and $J\left(C_{f}\right)$ its jacobian. Let $K^{\prime}$ be a finite algebraic extension of $K$.

Then for all primes $\ell$ and on each self-product $J\left(C_{f}\right)^{m}$ of $J\left(C_{f}\right)$ every $\ell$-adic Tate class over $K^{\prime}$ can be presented as a linear combination of products of divisor classes. In particular, the Tate conjecture holds true for all $J\left(C_{f}\right)^{m}$. 
Proof. Recall [33] that one may view Tate classes on $J\left(C_{f}\right)^{m}$ as tensor invariants of the Lie algebra $\mathfrak{g}_{\ell, J\left(C_{f}\right)} \cap \mathfrak{s p}\left(V_{\ell}\left(J\left(C_{f}\right), e_{\lambda}\right)\right.$ in

$$
V_{m, i}:=\operatorname{Hom}_{\mathbb{Q}_{\ell}}\left(\wedge_{\mathbb{Q}_{\ell}}^{2 i}\left(V_{\ell}\left(J\left(C_{f}\right)\right)^{m}\right), \mathbb{Q}_{\ell}\right) .
$$

By Theorem 8.3,

$$
\begin{aligned}
\mathfrak{g}_{\ell, J\left(C_{f}\right)} \bigcap \mathfrak{s p}\left(V_{\ell}\left(J\left(C_{f}\right)\right), e_{\lambda}\right) & = \\
{\left[\mathbb{Q}_{\ell} \operatorname{Id} \oplus \mathfrak{s p}\left(V_{\ell}\left(J\left(C_{f}\right)\right), e_{\lambda}\right)\right] \bigcap \mathfrak{s p}\left(V_{\ell}\left(J\left(C_{f}\right)\right), e_{\lambda}\right) } & =\mathfrak{s p}\left(V_{\ell}\left(J\left(C_{f}\right)\right), e_{\lambda}\right) .
\end{aligned}
$$

The invariant theory for symplectic groups ([13, Th. 2 on p. 543], [25]; see also [39]) tells us that every $\mathfrak{s p}\left(V_{\ell}\left(J\left(C_{f}\right)\right), e_{\lambda}\right)$-invariant in $V_{m, i}$ could be presented as a linear combination of exterior products of $\mathfrak{s p}\left(V_{\ell}\left(J\left(C_{f}\right)\right), e_{\lambda}\right)$-invariants in $V_{m, 1}$, i.e., of $\mathfrak{s p}\left(V_{\ell}\left(J\left(C_{f}\right)\right), e_{\lambda}\right)$-invariant alternating bilinear forms on

$$
V_{\ell}\left(J\left(C_{f}\right)\right)^{m}=\bigoplus_{j=1}^{m} V_{\ell}\left(J\left(C_{f}\right)\right) .
$$

The description of those alternating invariant bilinear forms given in Remark 8.1 implies that they all are linear combinations of divisors classes on $\left.J\left(C_{f}\right)\right)^{m}$ with coefficients in $\mathbb{Q}_{\ell}$. It follows that each $\ell$-adic Tate class can be presented as a a linear combination of products of divisor classes and therefore is algebraic.

Remark 9.2. In codimension 1 the Tate conjecture for all abelian varieties over $K$ is proven by Faltings $[9,10]$.

\section{Hodge Classes}

Let $X$ be a complex abelian variety of positive dimension, Let

$$
V_{\mathbb{Q}}=V_{\mathbb{Q}}(X):=\mathbb{H}_{1}(X(\mathbb{C}), \mathbb{Q})
$$

be the first rational homology group of the complex torus $X(\mathbb{C})$ and let

$$
e_{\lambda, \mathbb{Q}}: V_{\mathbb{Q}}(X) \times V_{\mathbb{Q}}(X) \rightarrow \mathbb{Q}
$$

be the alternating nondegenerate $\mathbb{Q}$-bilinear (Riemann) form attached to a polarization $\lambda$ on $X$. Let $\operatorname{Sp}\left(V_{\mathbb{Q}}(X), e_{\lambda, \mathbb{Q}}\right) \subset \mathrm{GL}\left(V_{\mathbb{Q}}(X)\right)$ be the $\mathbb{Q}$-algebraic symplectic group attached to $e_{\lambda, \mathbb{Q}}$. We write $\mathfrak{s p}\left(\left(V_{\mathbb{Q}}(X), e_{\lambda, \mathbb{Q}}\right)\right.$ for the Lie algebra of $\operatorname{Sp}\left(V_{\mathbb{Q}}(X), e_{\lambda, \mathbb{Q}}\right)$ : it is an absolutely simple absolutely irreducible $\mathbb{Q}$-Lie subalgebra of $\operatorname{End}_{\mathbb{Q}}\left(V_{\mathbb{Q}}(X)\right)$. Let $\mathrm{Gp}\left(V_{\mathbb{Q}}(X), e_{\lambda, \mathbb{Q}}\right) \subset \mathrm{GL}\left(V_{\mathbb{Q}}(X)\right)$ be the (connected) $\mathbb{Q}$ algebraic (sub)group of symplectic similitudes attached to $e_{\lambda, \mathbb{Q}}$. We have

$$
\operatorname{Sp}\left(V_{\mathbb{Q}}(X), e_{\lambda, \mathbb{Q}}\right) \subset \mathrm{Gp}\left(V_{\mathbb{Q}}(X), e_{\lambda, \mathbb{Q}}\right) \subset \mathrm{GL}\left(V_{\mathbb{Q}}(X)\right) .
$$

The Lie algebra of $\operatorname{Gp}\left(V_{\mathbb{Q}}(X), e_{\lambda, \mathbb{Q}}\right)$ coincides with $\mathbb{Q} \operatorname{Id} \oplus \mathfrak{s p}\left(\left(V_{\mathbb{Q}}(X), e_{\lambda, \mathbb{Q}}\right)\right.$; here Id is the identity map on $V_{\mathbb{Q}}(X)$.

We refer to [25] for the definition of the Mumford-Tate group $\mathrm{MT}=\mathrm{MT}_{X}$ of $X$; it is a reductive connected $\mathbb{Q}$-algebraic subgroup of $\operatorname{Gp}\left(V_{\mathbb{Q}}(X), e_{\lambda, \mathbb{Q}}\right)$. We have

$$
\operatorname{MT}_{X} \subset \operatorname{Gp}\left(V_{\mathbb{Q}}(X), e_{\lambda, \mathbb{Q}}\right) \subset \mathrm{GL}\left(V_{\mathbb{Q}}(X)\right) .
$$

We write $\mathrm{mt}_{X}$ for the Lie algebra of $\mathrm{MT}_{X}$ : it is a reductive algebraic $\mathbb{Q}$-Lie subalgebra of $\operatorname{End}_{\mathbb{Q}}\left(V_{\mathbb{Q}}(X)\right)$. It is well-known $[25]$ that

$$
\mathbb{Q} \operatorname{Id} \subset \mathrm{mt}_{X} \subset \mathbb{Q} \operatorname{Id} \oplus \mathfrak{s p}\left(V_{\mathbb{Q}}(X), e_{\lambda, \mathbb{Q}}\right) \subset \operatorname{End}_{\mathbb{Q}}\left(V_{\mathbb{Q}}(X)\right) .
$$

We refer to [29, Sect. 3 and 4$]$ for the precise statement and a discussion of the Mumford-Tate conjecture for abelian varieties. (See also [37].) 
Theorem 10.1. Suppose that $f(x) \in \mathbb{C}[x]$ is a polynomial of degree $n \geq 10$ without multiple roots. Let $C_{f}$ be the hyperelliptic curve $y^{2}=f(x)$ and $X=J\left(C_{f}\right)$ its jacobian, viewed as a complex abelian variety provided with the canonical principal polarization $\lambda$ attached to the theta divisor. Let

$$
e_{\lambda, \mathbb{Q}}: V_{\mathbb{Q}}(X) \times V_{\mathbb{Q}}(X) \rightarrow \mathbb{Q}
$$

be the alternating nondegenerate $\mathbb{Q}$-bilinear (Riemann) form attached to $\lambda$.

Suppose that all the coefficients of $f(x)$ lie in a subfield $K \subset \mathbb{C}$ and $f(x)=$ $(x-t) h(x)$ with $t \in K, h(x) \in K[x]$. Assume also that $K$ is finitely generated over $\mathbb{Q}$ and the Galois group of $h(x)$ over $K$ is either $\mathbf{S}_{n-1}$ or $\mathbf{A}_{n-1}$.

Then:

- The Mumford-Tate group of $X$ coincides with $\operatorname{Gp}\left(V_{\mathbb{Q}}(X), e_{\lambda, \mathbb{Q}}\right)$.

- Each Hodge class on every self-product $X^{m}$ of $X$ can be presented as a linear combination of products of divisor classes. In particular, the Hodge conjecture holds true for all $X^{m}$.

- The Mumford-Tate conjecture holds true for $J\left(C_{f}\right)$. (Here $J\left(C_{f}\right)$ is viewed as an abelian variety over $K$.)

Proof. We use the arguments from [42, p. 429]. Let $\bar{K} \subset \mathbb{C}$ be the algebraic closure of $K$ in $\mathbb{C}$. For each prime $\ell$ let us consider the $\mathbb{Q}_{\ell}$-vector space

$$
\Pi_{\ell}=V_{\mathbb{Q}}(X) \otimes_{\mathbb{Q}} \mathbb{Q}_{\ell} .
$$

Then there is a well-known (comparison) isomorphism of $\mathbb{Q}_{\ell}$-vector spaces $[20,38]$

$$
\gamma_{\ell}: \Pi_{\ell} \cong V_{\ell}\left(J\left(C_{f}\right)\right)
$$

such that, by a theorem of Piatetski-Shapiro-Deligne-Borovoi [5, 29],

$$
\gamma_{\ell} \mathfrak{g}_{\ell, X} \gamma_{\ell}^{-1} \subset \mathrm{mt}_{X} \otimes_{\mathbb{Q}} \mathbb{Q}_{\ell} \subset\left[\mathbb{Q} \operatorname{Id} \oplus \mathfrak{s p}\left(\left(V_{\mathbb{Q}}(X), e_{\lambda, \mathbb{Q}}\right)\right] \otimes_{\mathbb{Q}} \mathbb{Q}_{\ell}\right.
$$

It follows from Theorem 8.3, the $\mathbb{Q}_{\ell}$-dimension of $\mathfrak{g}_{\ell, X}$ and the $\mathbb{Q}$-dimension of $\mathbb{Q} \operatorname{Id} \oplus \mathfrak{s p}\left(V_{\mathbb{Q}}(X), e_{\lambda, \mathbb{Q}}\right)$ do coincide. It follows that

$$
\gamma_{\ell} \mathfrak{g}_{\ell, X} \gamma_{\ell}^{-1}=\operatorname{mt}_{X} \otimes_{\mathbb{Q}} \mathbb{Q}_{\ell}=\left[\mathbb{Q} \operatorname{Id} \oplus \mathfrak{s p}\left(V_{\mathbb{Q}}(X), e_{\lambda, \mathbb{Q}}\right)\right] \otimes_{\mathbb{Q}} \mathbb{Q}_{\ell} .
$$

The first equality means that the Mumford-Tate conjecture holds true for $J\left(C_{f}\right)$. The second equality means that the $\mathbb{Q}$-dimensions of $\operatorname{mt}_{X}$ and $\mathbb{Q} \operatorname{Id} \oplus \mathfrak{s p}\left(V_{\mathbb{Q}}(X), e_{\lambda, \mathbb{Q}}\right)$ do coincide. Since $\operatorname{mt}_{X} \subset \mathbb{Q} \operatorname{Id} \oplus \mathfrak{s p}\left(V_{\mathbb{Q}}(X), e_{\lambda, \mathbb{Q}}\right)$, we conclude that

$$
\mathrm{mt}_{X}=\mathbb{Q} \operatorname{Id} \oplus \mathfrak{s p}\left(V_{\mathbb{Q}}(X), e_{\lambda, \mathbb{Q}}\right) .
$$

This implies that $\mathrm{MT}_{X}=\operatorname{Gp}\left(V_{\mathbb{Q}}(X), e_{\mathbb{Q}}\right)$, because their $\mathbb{Q}$-Lie algebras do coincide.

Recall [25] that the Hodge classes on a self-product $X^{m}$ of $X$ can be viewed as tensor invariants of the $\mathbb{Q}$-Lie algebra

$$
\begin{aligned}
\operatorname{mt}_{X} \bigcap \mathfrak{s p}\left(V_{\mathbb{Q}}(X), e_{\lambda, \mathbb{Q}}\right)=\left[\mathbb{Q} \operatorname{Id} \oplus \mathfrak{s p}\left(V_{\mathbb{Q}}(X), e_{\lambda, \mathbb{Q}}\right)\right] \bigcap \mathfrak{s p}\left(V_{\mathbb{Q}}(X), e_{\lambda, \mathbb{Q}}\right)= \\
\\
\mathfrak{s p}\left(V_{\mathbb{Q}}(X), e_{\lambda, \mathbb{Q}}\right)
\end{aligned}
$$

in the $\mathbb{Q}$-vector space

$$
V_{\mathbb{Q}, m, i}:=\operatorname{Hom}_{\mathbb{Q}}\left(\wedge_{\mathbb{Q}}^{2 j}\left(V_{\mathbb{Q}}(X)^{m}\right), \mathbb{Q}\right)
$$

where

$$
V_{\mathbb{Q}}(X)^{m}:=\bigoplus_{j=1}^{m} V_{\mathbb{Q}}(X) .
$$


The invariant theory for symplectic groups ([13, Th. 2 on p. 543]) implies that every $\mathfrak{s p}\left(V_{\mathbb{Q}}\left(J\left(C_{f}\right)\right), e_{\lambda, \mathbb{Q}}\right)$-invariant tensor in $V_{\mathbb{Q}, m, i}$ could be presented as a linear combination of exterior products of $\mathfrak{s p}\left(V_{\mathbb{Q}}\left(J\left(C_{f}\right)\right), e_{\lambda, \mathbb{Q}}\right)$-invariants in $V_{\mathbb{Q}, m, 1}$, i.e., of $\mathfrak{s p}\left(V_{\ell}\left(J\left(C_{f}\right)\right), e_{\lambda, \mathbb{Q}}\right)$-invariant alternating bilinear forms on

$$
V_{\mathbb{Q}}\left(J\left(C_{f}\right)\right)^{m}=\bigoplus_{j=1}^{m} V_{\mathbb{Q}}\left(J\left(C_{f}\right)\right) .
$$

This implies [25] that each Hodge class on $X^{m}$ can be presented as a linear combination of products of divisor classes. In particular, every Hodge class on $X^{m}$ is algebraic, i.e., the Hodge conjecture is true for $X^{m}$ in all dimensions.

\section{Appendix}

\section{Corrigendum to [42]}

Page 408, Proposition 3.3(i), the first sentence. In addition, $\pi^{\prime}(H)$ is normal in $\pi^{\prime}(G)$ and $H^{\prime}$ is normal in $G^{\prime}$.

Page 409, line 3 . The $R_{j}$ should be $S_{j}$.

Page 409, Corollary 3.4(ii). In addition, $H_{-1, \alpha}$ is normal is $G_{-1, \alpha}$.

Page 421, Step 4. The $\operatorname{Ad}_{i, E}$ is the canonical central isogeny $\mathfrak{G}_{i} \rightarrow \mathfrak{G}_{i}^{\text {Ad }}$ of absolutely simple $E$-algebraic groups.

\section{Corrigendum to $[46]$}

Theorem 1.1 on page 408. The $B(h)$ in assertion (i) should be $B(f)$.

Lemma 3.5 on page 419. The $\operatorname{End}^{0}(Y)$ should be $\operatorname{End}^{0}(X)$ and the $Y$ should be $X$.

Theorem 3.11 on page 422 . The $Y$ should satisfy either condition (i) or condition (ii) (not necessarily both).

Proof of Theorem 3.11 on page 423 should be modified as follows. The assertion (in the last sentence of the first paragraph) that $\operatorname{Aut}(X)$ is a finite cyclic group does not follow from Theorem 3.7. However, every finite subgroup of $\operatorname{Aut}(X)$ is cyclic, because $\operatorname{Aut}(X) \subset \operatorname{End}^{0}(X)^{*}$ and, by Theorem 3.7, $\operatorname{End}^{0}(X)$ is a field. On the other hand, since the isomorphism $u$ is always defined over a finite Galois extension of the ground field $F$, the image of the cocycle-homomorphism $c$ is a finite subgroup of $\operatorname{Aut}(X)$ and therefore is a (finite) cyclic group. The rest of the proof remains unchanged.

Page 423: in the (third) displayed formula in the middle of page (the subscript) $Y$ should be $X$, as well as in the formula in the previous line.

Proof of Theorem 1.1 on page 429 . The $B(h)$ in the first displayed formula should be $B(f)$. The $G_{B(h)}$ in the next line should be $G_{B(f)}$.

\section{REFERENCES}

[1] F.A. Bogomolov, Sur l'algébricité des représentations $\ell$-adiques. C. R. Acad. Sci. Paris Sér. A-B 290 (1980), A701-A703.

[2] F.A. Bogomolov, Points of finite order on abelian varieties. Izv. Akad. Nauk SSSR Ser. Mat. 44 (1980), 782-804; Math. USSR Izv. 17 (1981), 55-72.

[3] N. Bourbaki, Groupes et algèbres de Lie, Chapitres VII et VIII. Hermann, Paris, 1975.

[4] J. H. Conway, R. T. Curtis, S. P. Norton, R. A. Parker, R. A. Wilson, Atlas of finite groups. Clarendon Press, Oxford, 1985.

[5] P. Deligne, Hodge cycles on abelian varieties (notes by J.S. Milne). In: Hodge cycles, motives, and Shimura varieties. Springer Lecture Notes in Mathematics, 900 (1982), pp. 9-100. 
[6] I. Dolgachev, D. Ortland, Point Sets in Projective Spaces and Theta Functions. Astérisque 165, 1988.

[7] J.S. Ellenberg, C. Elsholtz, C. Hall, E. Kowalski, Non-simple abelian varieties in a family: geometric and analytic approaches. J. London Math. Soc., to appear; arXiv:0804.2166 [math.NT].

[8] A. Elkin, Yu. G. Zarhin, Endomorphism algebras of hyperelliptic Jacobians and finite projective lines. arXiv:math.AG/0508120 .

[9] G. Faltings, Endlichkeitssätze für abelsche Varietäten über Zahlkörpern. Invent. Math. 73 (1983), 349-366; Erratum, 75 (1984), 381.

[10] G. Faltings, Complements to Mordell. In: Rational points (G. Faltings, G. Wüstholz, eds.) Second edition. Aspects of Mathematics, E6. Friedr. Vieweg \& Sohn, Braunschweig, 1986.

[11] W. Feit, J. Tits, Projective representations of minimum degree of group extensions. Canad. J. Math. 30 (1978), 1092-1102.

[12] P. Griffiths, J. Harris, Principles of algebraic geometry. John Wiley \& Sons, New York Chichester Brisbane Toronto, 1978.

[13] R. Howe, Remarks on classical invariant theory. Trans. Amer. Math. Soc. 313 (1989), 539570; Erratum, 318 (1990), 823.

[14] G. James, A. Kerber, The representation theory of symmetric groups, Encyclopedia of Mathematics and its Applications 16. Addison-Wesley, Reading, MA, 1981.

[15] N. Jacobson, Lie Algebras, Dover Publications, Inc., New York, 1979.

[16] P. B. Kleidman, M. W. Liebeck, On a theorem of Feit and Tits. Proc. Amer. Math. Soc. 107 (1989), 315-322.

[17] A.S. Kleshchev, Pham Huu Tiep, On restrictions of modular spin representations of symmetric and alternating groups. Trans. Amer. Math. Soc. 356 (2004), 1971-1999.

[18] D.W.Masser, Specializations of endomorphism rings of abelian varieties. Bull. Soc. Math. France 124 (1996), 457-476.

[19] B. Mortimer, The modular permutation representations of the known doubly transitive groups. Proc. London Math. Soc. (3) 41 (1980), 1-20.

[20] D. Mumford, Abelian varieties, 2nd edn, Oxford University Press, 1974.

[21] F. Oort, Endomorphism algebras of abelian varieties. In: Algebraic Geometry and Commutative Algebra in Honor of M. Nagata (Ed. H. Hijikata et al), Kinokuniya Cy, Tokyo 1988; Vol. II, pp. 469 - 502.

[22] D.S. Passman, Permutation groups. W.A. Benjamin, Inc., New York Amsterdam, 1968.

[23] R. Pink, $\ell$-adic algebraic monodromy groups, cocharacters, and the Mumford-Tate conjecture. J. reine angew. Math. 495 (1998), 187-237.

[24] K.A. Ribet, Calois action on division points of abelian varieties with real multiplication. Amer. J. Math. 98 (1976), 751-804.

[25] K.A. Ribet, Hodge classes on certain types of abelian varieties. Amer. J. Math. 105 (1983), 523-538.

[26] A. Silverberg, Fields of definition for homomorphisms of abelian varieties. J. Pure Applied Algebra 77 (1992), 253-262.

[27] J.-P. Serre, Sur les groupes de Galois attachés aux groupes p-divisibles. In: Proc. Conf. Local Fields (Driebergen, 1966). Springer Verlag, Berlin, 1967, 118-131. (E, vol. II, 72, pp. 325-338.)

[28] J.-P. Serre, Groupes algébriques associés aux modules de Hodge-Tate. Journées de Géométrie Algébrique de Rennes. (Rennes, 1978), Vol. III, pp. 155-188, Astérisque, 65, Soc. Math. France, Paris, 1979. (E, vol. II, 119, pp. 469-502.)

[29] J.-P. Serre, Représentations $\ell$-adiques. In: Algebraic number theory (Kyoto Internat. Sympos., Res. Inst. Math. Sci., Univ. Kyoto, Kyoto, 1976), pp. 177-193. Japan Soc. Promotion Sci., Tokyo, 1977. (E, vol. III, 112, pp. 384-400.)

[30] J.-P. Serre, Abelian $\ell$-adic representations and elliptic curves, 2nd edition. Addison-Wesley, 1989.

[31] J.-P. Serre, Lie algebras and Lie groups, 2nd edition. Springer Lecture Notes in Mathematics, 1992.

[32] I. Schur, Über die Darstellung der symmetrischen und der alternierenden Gruppen durch gebrochene lineare Substitutionen. J. reine angew. Math. 139 (1911), 155 - 250; Gesammelte Abhandlungen, Band I, 346-441, Springer, Berlin, 1973. 
[33] J.T. Tate, Algebraic cycles and poles of zeta functions. In: Arithmetical algebraic geometry, pp. 93-110, Harper \& Row, New York, 1965.

[34] T.A. Springer, Linear algebraic groups, 2nd edition. Birkhäuser, Boston, 1998.

[35] A. Wagner, The faithful linear representations of least degree of $\mathbf{S}_{n}$ and $\mathbf{A}_{n}$ over a field of characteristic 2. Math. Z. 151 (1976), 127-137.

[36] A. Wagner, An observation of the degrees of projective representations of the symmetric and alternating group over an arbitrary field. Arch. Math. 29 (1977), 583-589.

[37] Yu. G. Zarhin, Weights of simple Lie algebras in the cohomology of algebraic varieties. Izv. Akad. Nauk SSSR Ser. Mat. 48 (1984), 264-304; Math. USSR-Izv. 24 (1985), 245-282.

[38] Yu. G. Zarhin, Abelian varieties having a reduction of K3 type. Duke Math. J. 65 (1992), $511-527$.

[39] Yu. G. Zarhin, The Tate conjecture for nonsimple abelian varieties over finite fields. In: Algebra and number theory (Essen, 1992) (J. Ritter, G.Frey, eds.), pp. 267-296, de Gruyter, Berlin, 1994.

[40] Yu. G. Zarhin, Hyperelliptic jacobians without complex multiplication. Math. Res. Letters 7 (2000), 123-132.

[41] Yu. G. Zarhin, Hyperelliptic jacobians and modular representations. In: Moduli of abelian varieties (eds. C. Faber, G. van der Geer and F. Oort). Progress in Math., vol. 195 (2001), Birkhäuser, pp. 473-490.

[42] Yu. G. Zarhin, Very simple 2-adic representations and hyperelliptic jacobians. Moscow Math. J. 2 (2002), 403-431.

[43] Yu. G. Zarhin, Homomorphisms of hyperelliptic Jacobians. In: Trudy Mat. Inst. Steklova 241 (2003), 90-104; Proc. Steklov Inst. Math. 241 (2003), 79-92.

[44] Yu. G. Zarhin, Non-supersingular hyperelliptic jacobians. Bull. Soc. Math. France 132 (2004), 617-634

[45] Yu. G. Zarhin, Homomorphisms of abelian varieties. In: "Arithmetic, Geometry and Coding Theory (AGCT 2003)" (Y. Aubry, G. Lachaud, eds.), Séminaires et Congrés 11 (2005), 189-215.

[46] Yu. G. Zarhin, Del Pezzo surfaces of degree 1 and Jacobians. Math. Ann. 340 (2008), 407-435.

[47] Yu. G. Zarhin, Homomorphisms of abelian varieties over finite fields, pp. 315- 343. In: Higherdimensional geometry over finite fields (D. Kaledin, Yu. Tschinkel, eds.), IOS Press, Amsterdam, 2008; arXiv:0711.1615 [math.AG].

[48] Yu. G. Zarhin, Endomorphisms of superelliptic jacobians. Math. Z. 261 (2009), 691-707, 709.

Department of Mathematics, Pennsylvania State University, University Park, PA 16802, USA

E-mail address: zarhin@math.psu.edu 